\title{
Asymptotic Dynamics of a Stochastic SIR Epidemic System Affected by Mixed Nonlinear Incidence Rates
}

\author{
Ping Han, Zhengbo Chang, and Xinzhu Meng (iD \\ College of Mathematics and Systems Science, Shandong University of Science and Technology, Qingdao 266590, China \\ Correspondence should be addressed to Xinzhu Meng; mxz721106@sdust.edu.cn
}

Received 10 January 2020; Revised 28 March 2020; Accepted 21 April 2020; Published 8 May 2020

Academic Editor: Diego R. Amancio

Copyright (C) 2020 Ping Han et al. This is an open access article distributed under the Creative Commons Attribution License, which permits unrestricted use, distribution, and reproduction in any medium, provided the original work is properly cited.

\begin{abstract}
This paper considers a stochastic SIR epidemic system affected by mixed nonlinear incidence rates. Using Markov semigroup theory and the Fokker-Planck equation, we explore the asymptotic dynamics of the stochastic system. We first investigate the existence of a positive solution and its uniqueness. Furthermore, we prove that the stochastic system has an asymptotically stable stationary distribution. In addition, the sufficient conditions for disease extinction are also obtained, which imply that the white noise can suppress and control the spread of infectious diseases. Finally, in order to illustrate the analytical results, we give some numerical simulations.
\end{abstract}

\section{Introduction}

In 1927, the threshold conditions for disease transmission were established by Kermack and McKendrick [1]. In recent decades, many studies have applied threshold theory to various epidemic systems, and there have been a large amount of results related to the dynamical behaviors for various models. In addition, nonlinear incidence rates are very significant and are used frequently in the dynamics of epidemic models [2-11]. Hethcote [12] introduced an SIR epidemic system affected by bilinear incidence rate $\lambda S I$ :

$$
\left\{\begin{array}{l}
\mathrm{d} S=(u-u S-\lambda S I) \mathrm{d} t \\
\mathrm{~d} I=(\lambda S I-u I-\eta I) \mathrm{d} t \\
\mathrm{~d} R=(\eta I-u R) \mathrm{d} t
\end{array}\right.
$$

where the parameters $u, \lambda$, and $\eta$ are positive constants. $S, I$, and $R$ represent the density of susceptible, infectious, and recovered individuals, respectively. Suppose that the recruitment rate is the same as the natural death rate, denoted by $u$. $\lambda$ is the transmission coefficient, $\eta$ represents the recovery rate, and $\lambda_{1} S I$ represents the bilinear incidence rate. Furthermore, some researchers [13] proposed SIR epidemic models with saturated incidence $(\lambda S I /(1+m I))$ similar to the following form:

$$
\left\{\begin{array}{l}
\mathrm{d} S=\left(u-u S-\frac{\lambda S I}{1+m I}\right) \mathrm{d} t, \\
\mathrm{~d} I=\left(\frac{\lambda S I}{1+m I}-u I-\eta I\right) \mathrm{d} t, \\
\mathrm{~d} R=(\eta I-u R) \mathrm{d} t,
\end{array}\right.
$$

where $m$ is a positive constant. $\left(\lambda_{2} S I /(1+m I)\right)$ represents the saturated incidence rate. Particularly, Liu et al. [14] studied an SIS epidemic system with general nonlinear incidence rate effect by employing Markov semigroup theory. Inspired by previous works, we consider that the infected person may have immunity to return to recovery class after being cured or return to the susceptible class again. And the epidemic affected by mixed nonlinear incidence rates is more realistic compared to epidemic affected by a single nonlinear incidence rate. Thus, when two different incidence rates (bilinear and saturated incidence rates) are considered at the same time, the following 
deterministic SIR epidemic system affected by two different nonlinear incidence rates is studied:

$$
\left\{\begin{array}{l}
\mathrm{d} S=\left[u-u S-p \lambda_{1} S I-(1-p) \frac{\lambda_{2} S I}{1+m I}\right] \mathrm{d} t+[(1-\alpha) \eta I] \mathrm{d} t, \\
\mathrm{~d} I=\left[p \lambda_{1} S I+(1-p) \frac{\lambda_{2} S I}{1+m I}-u I-\eta I\right] \mathrm{d} t, \\
\mathrm{~d} R=(\alpha \eta I-u R) \mathrm{d} t
\end{array}\right.
$$

where the parameters $\alpha, \lambda_{1}, \lambda_{2}$, and $p$ are positive constants. $\alpha$ denotes the probability that the infected returns to recovery class, $1-\alpha$ denotes the probability that the infected returns to susceptible class correspondingly, $\lambda_{1}$ and $\lambda_{2}$ are the transmission coefficients, $p$ represents the probability that bilinear incidence rate occurs, and $1-p$ represents the probability that saturated incidence rate occurs correspondingly. The basic reproduction number $R_{0}=\left(\left(p \lambda_{1}+\right.\right.$ $\left.\left.(1-p) \lambda_{2}\right) /(u+\eta)\right)$ is the threshold of model (3).

However, the population systems with stochastic effect present some complex dynamics, which attracts the attention of widespread researchers [15-18]. There are many kinds of noise in the environment, among which the white noise is a relatively stable noise in the process of propagation. It has been widely used and studied in physics and has a relatively complete system in mathematical analysis and application. In order to consider a more realistic disease model and make it more practical to study the influence of environmental noise on infectious diseases, the white noise is used. There is a long history of using the white noise to depict the influence of environmental randomness on the spread of disease; some researchers $[19,20]$ put forward that the environmental white noise disturbs the system parameters stochastically, and parameter $\lambda_{i}(i=1,2)$ is an important parameter for the spread of disease. The researchers [21-29] investigated the effect of environment on the dynamic behaviors by introducing stochastic perturbation into deterministic models. Based on the discussion above, we assume that $\lambda_{i} \longrightarrow \lambda_{i}+\sigma_{i} \dot{B}_{i}(t)$, where $B_{i}(t)$ represents a standard Brownian motion with intensity $\sigma_{i}>0(i=1,2)$. Now, the stochastic SIR epidemic system corresponding to system (3) is as follows:

$$
\left\{\begin{array}{l}
\mathrm{d} S=\left[u-u S-p \lambda_{1} S I-(1-p) \frac{\lambda_{2} S I}{1+m I}\right] \mathrm{d} t+[(1-\alpha) \eta I] \mathrm{d} t-p \sigma_{1} S I \mathrm{~d} B_{1}(t)-(1-p) \frac{\sigma_{2} S I}{1+m I} \mathrm{~d} B_{2}(t) \\
\mathrm{d} I=\left[p \lambda_{1} S I+(1-p) \frac{\lambda_{2} S I}{1+m I}-u I-\eta I\right] \mathrm{d} t+p \sigma_{1} S I \mathrm{~d} B_{1}(t)+(1-p) \frac{\sigma_{2} S I}{1+m I} \mathrm{~d} B_{2}(t) \\
\mathrm{d} R=(\alpha \eta I-u R) \mathrm{d} t .
\end{array}\right.
$$

Since $\mathrm{d}(S+I+R)=[u-u(S+I+R)] \mathrm{d} t$, for any initial value $(S(0), I(0), R(0)) \in \mathbb{R}_{+}^{3}$ and $S(0)+I(0)+R(0)=1$, we always have $S(t)+I(t)+R(t)=1$. Therefore, $(S(t), I(t)$, $\left.R(t) \in \mathbb{R}_{+}^{3} \mid S(t)+I(t)+R(t)=1\right)$ is a positive invariant set of system (4). For simplicity, we let $R(t)=1-S(t)-I(t)$; then, the dynamics of system (4) is equivalent to the following two-dimensional system:

$$
\left\{\begin{aligned}
\mathrm{d} S= & {\left[u-u S-p \lambda_{1} S I-(1-p) \frac{\lambda_{2} S I}{1+m I}\right] \mathrm{d} t+[(1-\alpha) \eta I] \mathrm{d} t-p \sigma_{1} S I \mathrm{~d} B_{1}(t) } \\
& -(1-p) \frac{\sigma_{2} S I}{1+m I} \mathrm{~d} B_{2}(t), \\
\mathrm{d} I= & {\left[p \lambda_{1} S I+(1-p) \frac{\lambda_{2} S I}{1+m I}-u I-\eta I\right] \mathrm{d} t+p \sigma_{1} S I \mathrm{~d} B_{1}(t)+(1-p) \frac{\sigma_{2} S I}{1+m I} \mathrm{~d} B_{2}(t) . }
\end{aligned}\right.
$$

Next, we study the dynamical behaviors of system (5) which are affected by mixed nonlinear incidence rates. Particularly, as the main purpose, we study the asymptotically stable stationary distribution and extinction of epidemic by establishing the corresponding sufficient conditions.

In this paper, $\left(\Omega, \mathscr{F},\{\mathscr{F}\}_{t>0}, \mathbb{P}\right)$ represents a complete probability space with a filtration $\left\{\mathscr{F}_{t}\right\}_{t \geq 0}$ satisfying the 
usual conditions (i.e., it is increasing and right continuous, while $\mathscr{F}_{0}$ contains all $\mathbb{P}$-null sets). Define $B(t)$ is on the probability space. Denote $\mathbb{R}_{+}^{2}=\left\{x_{i}>0, i=1,2\right\}$.

\section{Preliminary Knowledge}

2.1. Markov Semigroups and Fokker-Planck Equation. In this section, we provide some definitions about Markov semigroups [14], asymptotic properties [30-34], and FokkerPlanck equation to verify our results.

Denote $\mathbb{X}, \Sigma$, and $m$ to be a metric space, $\sigma$-algebra of Borel sets, and the Lebesgue measure on $(\mathbb{X}, \Sigma)$, respectively. $\mathbb{D}=s\left\{h \in L^{1}: h \geq 0,\|h\|=1\right\} .\{P(t)\}_{t \geq 0}$ represents an integral Markov semigroup with a continuous kernel $\mathscr{K}(t ; x ; y)$ for $t>0$, which satisfies $\int_{\mathbb{X}} \mathscr{K}(x, y) m(\mathrm{~d} x)=1$ for $\forall y \in \mathbb{X}$. For the diffusion process $(S(t), I(t))$, i.e., the transition probability function is $\mathscr{P}(t, x, y, A)=\operatorname{Prob}\{(S(t), I(t)) \in A\}$. Define $\varphi_{1}(x, y)=x^{2} y^{2}, \varphi_{2}(x, y)=\left(x^{2} y^{2} /(1+m y)^{2}\right)$, and

$$
\left\{\begin{array}{l}
f_{1}(x, y)=u-u x-p \lambda_{1} x y-(1-p) \frac{\lambda_{2} x y}{1+m y}+(1-\alpha) \eta y, \\
f_{2}(x, y)=p \lambda_{1} x y+(1-p) \frac{\lambda_{2} x y}{1+m y}-u y-\eta y .
\end{array}\right.
$$

Remark 1. If for $\forall h \in \mathbb{D}$, we have

$$
\int_{0}^{\infty} P(t) h \mathrm{~d} t>0, \quad \text { a.e. }
$$

Then, this semigroup is asymptotically stable or sweeping with respect to compact sets $[30,31]$; this property is called the Foguel alternative [34].

Remark 2. If the distribution of $(S(t), I(t))$ is absolutely continuous with respect to the Lebesgue measure with the density $U(t, x, y)$, then $U$ satisfies the Fokker-Planck equation [32]:

$$
\begin{aligned}
\frac{\partial U}{\partial t}= & \frac{\sigma_{1}^{2} p^{2}}{2}\left(\frac{\partial^{2}\left(\varphi_{1} U\right)}{\partial x^{2}}-2 \frac{\partial^{2}\left(\varphi_{1} U\right)}{\partial x \partial y}+\frac{\partial^{2}\left(\varphi_{1} U\right)}{\partial y^{2}}\right) \\
& +\frac{\sigma_{2}^{2}(1-p)^{2}}{2}\left(\frac{\partial^{2}\left(\varphi_{2} U\right)}{\partial x^{2}}-2 \frac{\partial^{2}\left(\varphi_{2} U\right)}{\partial x \partial y}+\frac{\partial^{2}\left(\varphi_{2} U\right)}{\partial y^{2}}\right) \\
& -\frac{\partial\left(f_{1} U\right)}{\partial x}-\frac{\partial\left(f_{2} U\right)}{\partial y} .
\end{aligned}
$$

Remark 3. Denote $P(t) V(x, y)=U(x, y, t)$ for $V \in \mathbb{D}$, and $\mathscr{A}$ represents the infinitesimal generator of semigroup $\{P(t)\}_{t \geq 0}$; then, we have

$$
\begin{aligned}
\mathscr{A}^{*} V= & \frac{(1-p)^{2} \sigma_{2}^{2} \varphi_{2}}{2}\left(\frac{\partial^{2}(V)}{\partial x^{2}}-2 \frac{\partial^{2}(V)}{\partial x \partial y}+\frac{\partial^{2}(V)}{\partial y^{2}}\right) \\
& +\frac{p^{2} \sigma_{1}^{2} \varphi_{1}}{2}\left(\frac{\partial^{2}(V)}{\partial x^{2}}-2 \frac{\partial^{2}(V)}{\partial x \partial y}+\frac{\partial^{2}(V)}{\partial y^{2}}\right) \\
& +\frac{\partial\left(f_{1} V\right)}{\partial x}+\frac{\partial\left(f_{2} V\right)}{\partial y} .
\end{aligned}
$$

2.2. Some Lemmas about the Asymptotically Stable Stationary Distribution. In this section, five lemmas are given to study the asymptotically stable stationary distribution of system (5).

Lemma 1. The transition probability function $\mathscr{P}\left(t, x_{0}\right.$, $\left.y_{0}, A\right)$ has a continuous density $\mathscr{K}\left(t, x, y ; x_{0}, y_{0}\right)$ with respect to the Lebesgue measure.

Proof. Denote

$$
\vec{a}(x, y)=\left[\begin{array}{l}
a_{1} \\
a_{2}
\end{array}\right]
$$

$$
\overrightarrow{b^{1}}(x, y)=\left[\begin{array}{c}
-p \sigma_{1} x y \\
p \sigma_{1} x y
\end{array}\right]
$$

$$
\overrightarrow{b^{2}}(x, y)=\left[\begin{array}{c}
-(1-p) \frac{\lambda_{2} x y}{1+m y} \\
(1-p) \frac{\lambda_{2} x y}{1+m y}
\end{array}\right]
$$

where $a_{1}=u-u x-p \lambda_{1} x y-(1-p) \quad\left(\lambda_{2} x y /(1+m y)\right)+$ $(1-\alpha) \eta y, \quad a_{2}=p \lambda_{1} x y+(1-p)\left(\lambda_{2} x y /(1+m y)\right)-u y-\eta y$, and $(x, y) \in \mathbb{R}_{+}^{2} \cdot\left[\vec{a}, \overrightarrow{b^{i}}\right](i=1,2)$ is defined as

$$
\left[\vec{a}, \overrightarrow{b^{i}}\right]_{j}(x)=\sum_{k=1}^{2}\left(a_{k} \frac{\partial b_{j}^{i}}{\partial x_{k}}()-b_{k}^{i} \frac{\partial a_{j}}{\partial x_{k}}(x)\right), \quad j=1,2 .
$$

Then,

$$
\left[\vec{a}, \overrightarrow{b^{i}}\right]=\left(\begin{array}{c}
a_{1} \frac{\partial b_{1}^{i}}{\partial x}-b_{1}^{i} \frac{a_{1}}{\partial x}+a_{2} \frac{\partial b_{1}^{i}}{\partial y}-b_{2}^{i} \frac{a_{1}}{\partial y} \\
a_{1} \frac{\partial b_{2}^{i}}{\partial x}-b_{1}^{i} \frac{a_{2}}{\partial x}+a_{2} \frac{\partial b_{2}^{i}}{\partial y}-b_{2}^{i} \frac{a_{2}}{\partial y}
\end{array}\right)
$$

Denote $b_{1}^{i}=-b_{2}^{i}$, and by direct calculation, we have 


$$
\begin{aligned}
\left|\overrightarrow{b^{1}}\left[\vec{a}, \overrightarrow{b^{1}}\right]\right| & =\left|\begin{array}{ll}
b_{1}^{1} & a_{1} \frac{\partial b_{1}^{1}}{\partial x}-b_{1}^{1} \frac{a_{1}}{\partial x}+a_{2} \frac{\partial b_{1}^{1}}{\partial y}-b_{2}^{1} \frac{a_{1}}{\partial y} \\
b_{2}^{1} & a_{1} \frac{\partial b_{2}^{1}}{\partial x}-b_{1}^{1} \frac{a_{2}}{\partial x}+a_{2} \frac{\partial b_{2}^{1}}{\partial y}-b_{2}^{1} \frac{a_{2}}{\partial y}
\end{array}\right| \\
& =\alpha \eta p^{2} \sigma_{1}^{2} x^{2} y^{2}>0, \\
\left|\overrightarrow{b^{2}}\left[\vec{a}, \overrightarrow{b^{2}}\right]\right| & =\left|\begin{array}{ll}
b_{1}^{2} & a_{1} \frac{\partial b_{1}^{2}}{\partial x}-b_{1}^{2} \frac{a_{1}}{\partial x}+a_{2} \frac{\partial b_{1}^{2}}{\partial y}-b_{2}^{2} \frac{a_{1}}{\partial y} \\
b_{2}^{2} & a_{1} \frac{\partial b_{2}^{2}}{\partial x}-b_{1}^{2} \frac{a_{2}}{\partial x}+a_{2} \frac{\partial b_{2}^{2}}{\partial y}-b_{2}^{2} \frac{a_{2}}{\partial y}
\end{array}\right| \\
& =\alpha \eta(1-p)^{2} \frac{\sigma_{2}^{2} x^{2} y^{2}}{(1+m y)^{2}}>0,
\end{aligned}
$$

which shows that $\overrightarrow{b^{i}}$ and $\left[\vec{a}, \overrightarrow{b^{i}}\right]$ are independent on $\mathbb{R}_{+}^{2}$. Thus, according to Hö rmander theorem (see [35]), $\mathscr{P}\left(t, x_{0}, y_{0}, A\right)$ has a continuous density $\mathscr{K}\left(t, x, y ; x_{0}, y_{0}\right)$ and $\mathscr{K} \in C^{\infty}\left((0, \infty) \times \mathbb{R}_{+}^{2} \times \mathbb{R}_{+}^{2}\right)$. The proof is completed. In addition, we can obtain that the semigroup $\{P(t)\}_{t \geq 0}$ is an integral Markov semigroup.

Lemma 2. For each $\left(x_{0}, y_{0}\right) \in E$ and $(x, y) \in E$, where supp $U_{*}=\left\{(x, y) \in \mathbb{R}_{+}^{2}:(u /(u+\alpha \eta))<x+y<1\right\}:=E$, there exists $T>0$ such that $\mathscr{K}\left(T, x, y ; x_{0}, y_{0}\right)>0$.

Proof. Fix a point $\left(x_{0}, y_{0}\right) \in \mathbb{R}_{+}^{2}$ and a continuous function $\phi \in L^{2}([0, T] ; \mathbb{R})$; the following system is first considered:

$$
\left\{\begin{array}{l}
x_{\phi}(t)=x_{0}+\int_{0}^{t}\left(f_{1}\left(x_{\phi}(s), y_{\phi}(s)\right)-p \sigma_{1} \phi x_{\phi}(s) y_{\phi}(s)\right) \mathrm{d} s-\int_{0}^{t}(1-p) \frac{\sigma_{2} \phi x_{\phi}(s) y_{\phi}(s)}{1+m y_{\phi}(s)} \mathrm{d} s \\
y_{\phi}(t)=y_{0}+\int_{0}^{t}\left(f_{2}\left(x_{\phi}(s), y_{\phi}(s)\right)+p \sigma_{1} \phi x_{\phi}(s) y_{\phi}(s)\right) \mathrm{d} s+\int_{0}^{t}(1-p) \frac{\sigma_{2} \phi x_{\phi}(s) y_{\phi}(s)}{1+m y_{\phi}(s)} \mathrm{d} s .
\end{array}\right.
$$

Step 1. Find $D_{x_{0}, y_{0} ; \phi}^{i}$ by the perturbation method. Denote $\mathbf{f}^{\prime}$, $\mathbf{g}^{\mathbf{1}^{\prime}}$, and $\mathbf{g}^{\mathbf{2}^{\prime}}$ are the Jacobians of

$$
\begin{aligned}
\mathbf{f} & =\left(\begin{array}{c}
f_{1}(x, y) \\
f_{2}(x, y)
\end{array}\right), \\
\mathbf{g}^{1} & =\left(\begin{array}{c}
-p \sigma_{1} x y \\
p \sigma_{1} x y
\end{array}\right), \\
\mathbf{g}^{2} & =\left(\begin{array}{c}
-(1-p) \frac{\sigma_{2} x y}{1+m y} \\
(1-p) \frac{\sigma_{2} x y}{1+m y}
\end{array}\right),
\end{aligned}
$$

respectively; then,

$$
\mathrm{F}^{i}=\mathbf{f}^{\prime}\left(x_{\phi}(t), y_{\phi}(t)\right)+\mathbf{g}^{\mathbf{i}^{\prime}}\left(x_{\phi}(t), y_{\phi}(t)\right) \phi
$$

For $0 \leq t_{0} \leq t \leq T$, let $Q^{i}\left(t_{0}, t_{0}\right)=I$ and $\left(\partial Q^{i}\left(t, t_{0}\right) / \partial t\right)=$ $\mathrm{F}^{i}(t) Q^{i}\left(t, t_{0}\right)$; we get

$$
D_{x_{0}, y_{0} ; \phi}^{i} h=\int_{0}^{T} Q^{i}(T, s) \mathbf{g}^{i}(s) h(s) \mathrm{d} s .
$$

Assume that the rank of $D_{x_{0}, y_{0} ; \phi}^{i}$ is 2 for some $\phi \in L^{2}([0, T] ; \mathbb{R})$; then, for $(x, y) \stackrel{x_{0}}{=}\left(x_{\phi}(T), y_{\phi}(T)\right)$, we have $\mathscr{K}\left(T, x, y ; x_{0}, y_{0}\right)>0$.
Step 2. Prove that the derivative $D_{x_{0}, y_{0} ; \phi}^{i}$ has rank 2. Replace system (14) with

$$
\left\{x_{\phi}^{\prime}(t)=y_{\phi}^{\prime}(t)=\left\{\begin{array}{l}
f_{1}\left(x_{\phi}(t), y_{\phi}(t)\right)-p \sigma_{1} \phi x_{\phi}(t) y_{\phi}(t) \\
-(1-p) \frac{\sigma_{2} \phi x_{\phi}(t) y_{\phi}(t)}{1+m y_{\phi}(t)} \\
f_{2}\left(x_{\phi}(t), y_{\phi}(t)\right)+p \sigma_{1} \phi x_{\phi}(t) y_{\phi}(t) \\
+(1-p) \frac{\sigma_{2} \phi x_{\phi}(t) y_{\phi}(t)}{1+m y_{\phi}(t)} .
\end{array}\right.\right.
$$

Define $\epsilon \in(0, T)$ and $h(t)=\left(\mathbf{1}_{[T-\epsilon, T]} / x_{\phi}(t) y_{\phi}(t)\right.$ $\left.f\left(x_{\phi}(t), y_{\phi}(t)\right)\right)$ for $t \in[0, T]$; here, $\mathbf{1}_{[T-\epsilon, T]}$ denotes the characteristic function of interval $[T-\epsilon, T]$. Using Taylor expansion, we obtain

$$
Q^{i}(T, s)=I+\mathrm{F}^{i}(T)(T-s)+o(T-s) .
$$

Then, we get

$$
\begin{aligned}
D_{x_{0}, y_{0} ; \phi}^{i} h & =\int_{0}^{T}\left[I+\mathrm{F}^{i}(T)(T-s)+o(T-s)\right] g^{i}(s) h(s) \mathrm{d} s \\
& =\epsilon \mathbf{v}+\frac{\epsilon^{2}}{2} \mathrm{~F}^{i}(T) \mathbf{v}+o\left(\epsilon^{2}\right),
\end{aligned}
$$

and $\mathbf{v}^{1}=\left(-\sigma_{1}, \sigma_{1}\right)^{\mathrm{T}}, \mathbf{v}^{2}=\left(-\sigma_{2}, \sigma_{2}\right)^{\mathrm{T}}$. 
Then,

$$
\begin{aligned}
\mathrm{F}^{1}(T) \mathbf{v}^{1} & =\sigma_{1}\left[\begin{array}{l}
A \\
B
\end{array}\right] \\
\left|\mathbf{v}^{1} \mathrm{~F}^{1}(T) \mathbf{v}^{1}\right| & =\left|\begin{array}{cc}
-\sigma_{1} & \sigma_{1} A \\
\sigma_{1} & \sigma_{1} B
\end{array}\right|=\sigma_{1}^{2} \alpha \eta>0, \\
\mathrm{~F}^{2}(T) \mathbf{v}^{2} & =\sigma_{2}\left[\begin{array}{l}
C \\
D
\end{array}\right] \\
\left|\mathbf{v}^{2} \mathrm{~F}^{2}(T) \mathbf{v}^{2}\right|\left|\begin{array}{cc}
-\sigma_{2} & \sigma_{2} C \\
\sigma_{2} & \sigma_{2} D
\end{array}\right| & =\sigma_{2}^{2} \alpha \eta>0
\end{aligned}
$$

where

$$
\begin{aligned}
A= & p\left(\lambda_{1}+\sigma_{1} \phi\right)(y-x) \\
& +(1-p) \lambda_{2} \frac{-x+y+m y^{2}}{(1+m y)^{2}}+(1-\alpha) \eta+u, \\
B= & p\left(\lambda_{1}+\sigma_{1} \phi\right)(x-y)+(1-p) \lambda_{2} \frac{x-y-m y^{2}}{(1+m y)^{2}}-(u+\eta), \\
C= & u+p \lambda_{1} x+(1-p) \frac{\lambda_{2} x}{(1+m y)^{2}}-(1-p) \frac{\sigma_{2} x}{(1+m y)^{2}}
\end{aligned}
$$

$$
\begin{aligned}
& +(1-\alpha) \eta-p \lambda_{1} y-(1-p) \frac{\lambda_{2} y}{1+m y} \\
& +(1-p) \frac{\sigma_{2} y}{1+m y}, \\
D= & -u-p \lambda_{1} x-(1-p) \frac{\lambda_{2} x}{(1+m y)^{2}}+(1-p) \frac{\sigma_{2} x}{(1+m y)^{2}} \\
& -\eta+p \lambda_{1} y+(1-p) \frac{\lambda_{2} y}{1+m y}-(1-p) \frac{\sigma_{2} y}{1+m y} .
\end{aligned}
$$

Therefore, the rank of $D_{x_{0}, y_{0} ; \phi}^{i}$ is 2 .

Step 3. Verify that, for any $\left(x_{0}, y_{0}\right) \in E$ and $(x, y) \in E$, there exist control function $\phi$ and $T>0$ such that $\left(x_{\phi}(0), y_{\phi}(0)\right)=$ $\left(x_{0}, y_{0}\right)$ and $\left(x_{\phi}(T), y_{\phi}(T)\right)=(x, y)$. Let $z_{\phi}=x_{\phi}+y_{\phi}$, and $\left\{\begin{array}{l}g_{1}(x, z)=u-u x-p \lambda_{1} x(z-x)-(1-p) \frac{\lambda_{2} x(z-x)}{1+m(z-x)}, \\ g_{2}(x, z)=u+\alpha \eta x-(u+\alpha \eta) z .\end{array}\right.$

System (18) becomes

$$
\left\{\begin{array}{l}
x_{\phi}^{\prime}(t)=g_{1}\left(x_{\phi}(t), z_{\phi}(t)\right)-p \sigma_{1} \phi x_{\phi}(t)\left(z_{\phi}(t)-x_{\phi}(t)\right)-(1-p) \frac{\sigma_{2} \phi x_{\phi}(t)\left(z_{\phi}(t)-x_{\phi}(t)\right)}{1+m\left(z_{\phi}(t)-x_{\phi}(t)\right)} \\
z_{\phi}^{\prime}(t)=g_{2}\left(x_{\phi}(t), z_{\phi}(t)\right) .
\end{array}\right.
$$

\section{Denote}

$$
E_{0}=\left\{(x, z) \in \mathbb{R}_{+}^{2}: 0<x<1, \frac{u}{u+\alpha \eta}<z<1, x<z\right\} .
$$

Using the same proof method of Lemma 3.2 in [36], we can obtain that, for any $\left(x_{0}, z_{0}\right) \in E_{0}$ and $(x, z) \in E_{0}$, there exist control function $\phi$ and $T>0$ such that $\left(x_{\phi}(0), z_{\phi}(0)\right)=$ $\left(x_{0}, z_{0}\right)$ and $\left(x_{\phi}(T), z_{\phi}(T)\right)=(x, z)$. Now, the positivity of $\mathscr{K}$ is proved by support theorems (see [37-39]).

Lemma 3. If $R_{0}-\left(\left(p^{2} \sigma_{1}^{2}+(1-p)^{2} \sigma_{2}^{2}\right) / 2(u+\eta)\right)>1$, for every density $f$ and the semigroup $\{P(t)\}_{t \geq 0}$, the following conclusion holds:

$$
\lim _{t \rightarrow \infty} \iint_{E} P(t) f(x, y) \mathrm{d} x \mathrm{~d} y=1 .
$$

Proof. Substitute $Z(t)=S(t)+I(t)$; we can obtain that

$$
u-(u+\alpha \eta) Z(t)<\frac{d Z(t)}{d t}<u-u Z(t), \quad t \in(0, \infty) \text { a.s. }
$$

Next, in the following three cases, (i) $Z(0) \in(0,(u /(u+\alpha \eta)))$, (ii) $Z(0) \in((u /(u+\alpha \eta)), 1)$, and (iii) $Z(0) \in(1, \infty)$, we verify that there is $t_{0}=t_{0}(\omega)$ such that

$$
\frac{u}{u+\alpha \eta}<Z(t, \omega)<1, \quad t>t_{0}
$$

for almost every $\omega \in \Omega$; this proof is completed.

(i) If inequality (28) is not true, then there exists $\Omega^{\prime} \subset \Omega$ with $\operatorname{Prob}\left(\Omega^{\prime}\right)>0$ which satisfies

$$
Z(t, \omega) \in\left(0, \frac{u}{u+\alpha \eta}\right)
$$

On the one hand, we can get that $(\mathrm{d} Z(t) / \mathrm{d} t)>u-$ $(u+\alpha \eta) Z(t)>0$ from $(27)$. Thus, $Z(t, \omega)$ is strictly increasing on $[0, \infty)$ for any $\omega \in \Omega^{\prime}$, and then 
$\lim _{t \rightarrow \infty} Z(t, \omega)=(u /(u+\alpha \eta))$. In view of the expression of $g_{2}$, we have

$$
\begin{aligned}
& \lim _{t \longrightarrow \infty} S(t, \omega)=0, \\
& \lim _{t \longrightarrow \infty} I(t, \omega)=\frac{u}{u+\alpha \eta} .
\end{aligned}
$$

Namely,

$$
\lim _{t \longrightarrow \infty} \frac{\ln I(t)-\ln I(0)}{t}=0, \quad \omega \in \Omega^{\prime} .
$$

On the other hand, according to Itô's formula, we get

$$
\begin{aligned}
\mathrm{d} \ln I(t)= & {\left[p \lambda_{1} S+(1-p) \frac{\lambda_{2} S}{1+m I}-u-\eta\right] \mathrm{d} t } \\
& -\left[\frac{1}{2}\left(p^{2} \sigma_{1}^{2} S^{2}+(1-p)^{2} \frac{\sigma_{2}^{2} S^{2}}{(1+m I)^{2}}\right)\right] \mathrm{d} t \\
& +p \sigma_{1} S \mathrm{~d} B_{1}(t)+(1-p) \frac{\sigma_{2} S}{1+m I} \mathrm{~d} B_{2}(t) .
\end{aligned}
$$

Therefore,

$$
\begin{aligned}
\frac{\ln I(t)-\ln I(0)}{t}= & \frac{1}{t} \int_{0}^{t} p \lambda_{1} S(s)+(1-p) \frac{\lambda_{2} S(s)}{1+m I(s)} \mathrm{d} s \\
& -\frac{1}{t} \int_{0}^{t} \frac{1}{2} p^{2} \sigma_{1}^{2} S^{2}(s) \mathrm{d} s \\
& -\frac{1}{t} \int_{0}^{t} \frac{1}{2}(1-p)^{2} \frac{\sigma_{2}^{2} S^{2}(s)}{(1+m I(s))^{2}} \mathrm{~d} s \\
& +\frac{1}{t} \int_{0}^{t}(-u-\eta) \mathrm{d} s+\frac{M_{1}(t)}{t}+\frac{M_{2}(t)}{t},
\end{aligned}
$$

where

$$
\begin{aligned}
M_{1}(t) & =p \sigma_{1} \int_{0}^{t} S(s) \mathrm{d} B_{1}(s), \\
M_{2}(t) & =(1-p) \sigma_{2} \int_{0}^{t} \frac{S(s)}{1+m I(s)} \mathrm{d} B_{2}(s), \\
\left\langle M_{1}, M_{1}\right\rangle_{t} & =p^{2} \sigma_{1}^{2} \int_{0}^{t} S^{2}(s) \mathrm{d} s, \\
\left\langle M_{2}, M_{2}\right\rangle_{t} & =(1-p)^{2} \sigma_{2}^{2} \int_{0}^{t}\left(\frac{S(s)}{1+m I(s)}\right)^{2} \mathrm{~d} s .
\end{aligned}
$$

Clearly,

$$
\begin{aligned}
\limsup _{t \rightarrow \infty} \frac{\left\langle M_{1}, M_{1}\right\rangle_{t}}{t}= & \limsup _{t \rightarrow \infty} \frac{p^{2} \sigma_{1}^{2} \int_{0}^{t} S^{2}(s) \mathrm{d} s}{t} \\
& <\frac{p^{2} \sigma_{1}^{2} \int_{0}^{t} 1^{2} \mathrm{~d} s}{t}<p^{2} \sigma_{1}^{2}<\infty, \quad \text { a.s. } \\
\limsup _{t \rightarrow \infty} \frac{\left\langle M_{2}, M_{2}\right\rangle_{t}}{t}= & \limsup _{t \rightarrow \infty} \frac{(1-p)^{2} \sigma_{2}^{2} \int_{0}^{t}((S(s) /(1+m I(s))))^{2} \mathrm{~d} s}{t} \\
& <\frac{(1-p)^{2} \sigma_{2}^{2} \int_{0}^{t} 1^{2} \mathrm{~d} s}{t}<(1-p)^{2} \sigma_{2}^{2} \\
& <\infty, \text { a.s. }
\end{aligned}
$$

By the strong law of large numbers (see [40]), we get

$$
\lim _{t \rightarrow \infty} \frac{M_{1}(t)}{t}=\lim _{t \rightarrow \infty} \frac{M_{2}(t)}{t}=0, \quad \text { a.s. }
$$

Namely, 


$$
\begin{aligned}
\lim _{t \rightarrow \infty} \frac{\ln I(t)-\ln I(0)}{t}= & \lim _{t \rightarrow \infty}\left(-u-\eta+\frac{p \lambda_{1} \int_{0}^{t} S(s) \mathrm{d} s}{t}\right) \\
& +\lim _{t \rightarrow \infty} \frac{(1-p) \lambda_{2} \int_{0}^{t}(S(s) /(1+m I(s))) \mathrm{d} s}{t} \\
& -\lim _{t \rightarrow \infty} \frac{p^{2} \sigma_{1}^{2} \int_{0}^{t} S^{2}(s) \mathrm{d} s}{2 t} \\
& -\lim _{t \rightarrow \infty} \frac{(1-p)^{2} \sigma_{2}^{2} \int_{0}^{t}(S(s) /(1+m I(s))) \mathrm{d} s}{2 t} \\
= & -(u+\eta), \quad \text { a.s. on } \Omega^{\prime},
\end{aligned}
$$

this contradiction arises. Hence, the result follows for $Z(0) \in(0,(u /(u+\alpha \eta)))$.

(ii) From (27), we obtain that

$$
\begin{aligned}
& Z(t)>\frac{u}{u+\alpha}+\left[\left(Z_{0}-\frac{u}{u+\alpha}\right) e^{(u+\alpha) t_{0}}\right] e^{-(u+\alpha) t}, \\
& Z(t)<1+\left[\left(Z_{0}-1\right) e^{u t_{0}}\right] e^{-u t}<Z(t),
\end{aligned}
$$

Clearly, conclusion (28) holds in the case of $Z(0) \in((u /(u+\alpha \eta)), 1)$.

(iii) Suppose that inequality (28) does not hold. On the one hand, using the same way to (ii), we have $\lim _{t \rightarrow \infty} Z(t, \omega)=1, \omega \in \Omega^{\prime}$, and

$$
\begin{aligned}
& \lim _{t \longrightarrow \infty} S(t, \omega)=1, \\
& \lim _{t \longrightarrow \infty} I(t, \omega)=0 .
\end{aligned}
$$

Clearly,

$$
\lim _{t \rightarrow \infty} \frac{\ln I(t)-\ln I(0)}{t}=0, \quad \omega \in \Omega \prime .
$$

On the other hand, if $R_{0}-1>\left(\left(p^{2} \sigma_{1}^{2}+(1-p)^{2} \sigma_{2}^{2}\right) /\right.$ $2(u+\eta))$, we obtain

$$
\begin{aligned}
& \frac{\ln I(t)-\ln I(0)}{t}= p \lambda_{1}+(1-p) \lambda_{2}-u-\eta \\
&-\frac{p^{2} \sigma_{1}^{2}+(1-p)^{2} \sigma_{2}^{2}}{2} \\
&=(u+\eta)\left[R_{0}-1-\frac{p^{2} \sigma_{1}^{2}+(1-p)^{2} \sigma_{2}^{2}}{2(u+\eta)}\right] \\
&>0, \quad \text { a.s. on } \Omega^{\prime},
\end{aligned}
$$

so there is a contradiction. Thus, the result holds for $Z(0) \in(1, \infty)$. This proof is completed.
Lemma 4. If $R_{0}-\left(\left(p^{2} \sigma_{1}^{2}+(1-p)^{2} \sigma_{2}^{2}\right) / 2(u+\eta)\right)>1$, the semigroup $\{P(t)\}_{t \geq 0}$ is asymptotically stable or sweeping with respect to compact sets.

Proof. Using the same proof method of Lemma 4.5 in [14], this means that our result holds. We omit it here.

Lemma 5. The semigroup $\{P(t)\}_{t>0}$ is asymptotically stable if $R_{0}-\left(p^{2} \sigma_{1}^{2}+(1-p)^{2} \sigma_{2}^{2} / 2(u+\eta)\right)>1$.

Proof. By system (5), we obtain that

$$
\begin{aligned}
L(-\ln I)= & -p \lambda_{1} S-(1-p) \frac{\lambda_{2} S}{1+m I}+u+\eta \\
& +\frac{p^{2} \sigma_{1}^{2} S^{2}+(1-p)^{2} \sigma_{2}^{2}((S /(1+m I)))^{2}}{2} \\
\leq & -p \lambda_{1} S-(1-p) \frac{\lambda_{2} S}{1+m I}+u+\eta \\
& +\frac{p^{2} \sigma_{1}^{2}+(1-p)^{2} \sigma_{2}^{2}}{2} \\
= & -p \lambda_{1} S-(1-p) \lambda_{2} S+u+\eta \\
& +\frac{p^{2} \sigma_{1}^{2}+(1-p)^{2} \sigma_{2}^{2}}{2}+(1-p) \lambda_{2} S \\
& -(1-p) \frac{\lambda_{2} S}{1+m I} \\
& +\frac{m I}{1+m I}(1-p) \lambda_{2} S . \\
& +\frac{p^{2} \sigma_{1}^{2}+(1-p)^{2} \sigma_{2}^{2}}{2} \\
& (1-p) \lambda_{2} S+u+\eta
\end{aligned}
$$

Denote 


$$
\left\{\begin{array}{l}
f_{1}(S, I)=u-u S-p \lambda_{1} S I-(1-p) \frac{\lambda_{2} S I}{1+m I}+(1-\alpha) \eta I, \\
f_{2}(S, I)=p \lambda_{1} S I+(1-p) \frac{\lambda_{2} S I}{1+m I}-u I-\eta I,
\end{array}\right.
$$

$\mathscr{A}^{*} V=F\left[\frac{\partial^{2}(V)}{\partial S^{2}}-2 \frac{\partial^{2}(V)}{\partial S \partial I}+\frac{\partial^{2}(V)}{\partial I^{2}}\right]+\frac{\partial\left(f_{1} V\right)}{\partial S}+\frac{\partial\left(f_{2} V\right)}{\partial I}$,

where $F=\left(S^{2} I^{2}\left[p^{2} \sigma_{1}^{2}+(1-p)^{2} \sigma_{2}^{2}((1 /(1+m I)))^{2}\right] / 2\right)$, and we call $V$ a Khasminskii function [41].

$\{P(t)\}_{t \geq 0}$ satisfies the Foguel alternative according to Lemma 4 . We can exclude sweeping by constructing a nonnegative $C^{2}$-function $V$ and a closed set $U \in \Sigma$ satisfying

$$
\sup _{(S, I) \in \mathbb{X} \mid U} \mathscr{A}^{*} V(S, I)<-1
$$

Step 1. Construct a nonnegative $C^{2}$-function $V$. For $(S, I) \in E$, define

$$
\begin{aligned}
g(S, I)= & G\left(-\ln I+\frac{p \lambda_{1}+(1-p) \lambda_{2}}{u}(-S-I)\right) \\
& -\ln S-\ln (1-S-I)-\ln \left(S+I-\frac{u}{u+\alpha \eta}\right),
\end{aligned}
$$

where $G>0$ such that

$$
\begin{aligned}
& -G(u+\eta)\left(R_{0}-1-\frac{p^{2} \sigma_{1}^{2}+(1-p)^{2} \sigma_{2}^{2}}{2(u+\eta)}\right)+3 u+\alpha \eta \\
& +\left(p \lambda_{1}+(1-p) \lambda_{2}\right)+\frac{p^{2} \sigma_{1}^{2}+(1-p)^{2} \sigma_{2}^{2}}{2} \leq-2 .
\end{aligned}
$$

Let

$$
\begin{aligned}
V(S, I)= & g(S, I)-g(\bar{S}, \bar{I}) \\
= & G\left(-\ln I+\frac{p \lambda_{1}+(1-p) \lambda_{2}}{u}(-S-I)\right)-\ln S \\
& -\ln (1-S-I)-\ln \left(S+I-\frac{u}{u+\alpha \eta}\right)-g(\bar{S}, \bar{I}) .
\end{aligned}
$$

Denote

$$
\begin{aligned}
& V_{1}=-\ln I+\frac{p \lambda_{1}+(1-p) \lambda_{2}}{u}(-S-I), \\
& V_{2}=-\ln S, \\
& V_{3}=-\ln (1-S-I), \\
& V_{4}=-\ln \left(S+I-\frac{u}{u+\alpha \eta}\right)-g(\bar{S}, \bar{I}) .
\end{aligned}
$$

Step 2. Denote a closed set $U \in \Sigma$. For a sufficiently small positive number $\varepsilon$, we denote

$$
U=\left\{(S, I) \in E: \varepsilon \leq S, \varepsilon \leq I, K_{1} \leq S+I \leq K_{2}\right\},
$$

where $K_{1}=(u /(u+\alpha \eta))+\varepsilon^{2}, K_{2}=1-\varepsilon^{2}$. In the set $E \backslash U$, let $\varepsilon$ be sufficiently small such that

$$
\begin{gathered}
G m \varepsilon(1-p) \lambda_{2}+\frac{G\left[p \lambda_{1}+(1-p) \lambda_{2}\right]}{u}(\alpha \eta+u) \varepsilon<1, \\
-\frac{u}{\varepsilon}+G m(1-p) \lambda_{2}+\frac{G\left[p \lambda_{1}+(1-p) \lambda_{2}\right]}{u}(\alpha \eta+u)+H<-1, \\
-\frac{\alpha \eta}{\varepsilon}+G m(1-p) \lambda_{2}+\frac{G\left[p \lambda_{1}+(1-p) \lambda_{2}\right]}{u}(\alpha \eta+u)+H<-1 .
\end{gathered}
$$

Step 3. Prove

$$
\sup _{(S, I) \in \mathbb{X} \backslash U} \mathscr{A}^{*} V(S, I)<-1,
$$

$$
\begin{aligned}
\mathscr{A}^{*} V_{1} \leq & -\left[p \lambda_{1}+(1-p) \lambda_{2}\right] S+u+\eta+\frac{p^{2} \sigma_{1}^{2}+(1-p)^{2} \sigma_{2}^{2}}{2} \\
& +\frac{m I}{1+m I}(1-p) \lambda_{2} S-\left[p \lambda_{1}+(1-p) \lambda_{2}\right] \\
& +\left[p \lambda_{1}+(1-p) \lambda_{2}\right] S+\frac{p \lambda_{1}+(1-p) \lambda_{2}}{u}(\alpha \eta+u) I \\
= & -\left[p \lambda_{1}+(1-p) \lambda_{2}\right]+u+\eta+\frac{p^{2} \sigma_{1}^{2}+(1-p)^{2} \sigma_{2}^{2}}{2} \\
& +\frac{m I}{1+m I}(1-p) \lambda_{2} S+\frac{p \lambda_{1}+(1-p) \lambda_{2}}{u}(\alpha \eta+u) I \\
\leq & -\left[p \lambda_{1}+(1-p) \lambda_{2}\right]+u+\eta+\frac{p^{2} \sigma_{1}^{2}+(1-p)^{2} \sigma_{2}^{2}}{2} \\
& +\frac{m I}{1+m I}(1-p) \lambda_{2}+\frac{p \lambda_{1}+(1-p) \lambda_{2}}{u}(\alpha \eta+u) I \\
:= & \frac{m I}{1+m I}(1-p) \lambda_{2}+\frac{p \lambda_{1}+(1-p) \lambda_{2}}{u}(\alpha \eta+u) I \\
& -\lambda,
\end{aligned}
$$

where $\quad \lambda=\left[p \lambda_{1}+(1-p) \quad \lambda_{2}\right]-u-\eta-\left(\left(p^{2} \sigma_{1}^{2}+(1-p)^{2}\right.\right.$ $\left.\left.\sigma_{2}^{2}\right) / 2\right)=(u+\eta)\left[R_{0}-1-\left(\left(p^{2} \sigma_{1}^{2}+(1-p)^{2} \sigma_{2}^{2}\right) / 2(u+\eta)\right)\right]>0$. 


$$
\begin{aligned}
\mathscr{A}^{*} V_{2}= & -\frac{u}{S}+u+p \lambda_{1}+(1-p) \frac{\lambda_{2} I}{1+m I}-\frac{(1-\alpha) \eta I}{S} \\
& +\frac{p^{2} \sigma_{1}^{2} I^{2}+(1-p)^{2}\left(\sigma_{2}^{2} I^{2} /(1+m I)^{2}\right)}{2} \\
\leq & -\frac{u}{S}+u+p \lambda_{1}+(1-p) \lambda_{2}+\frac{p^{2} \sigma_{1}^{2}+(1-p)^{2} \sigma_{2}^{2}}{2} \\
= & -\frac{u}{S}+\left[p \lambda_{1}+(1-p) \lambda_{2}\right]+u+\frac{p^{2} \sigma_{1}^{2}+(1-p)^{2} \sigma_{2}^{2}}{2} \\
\mathscr{A}^{*} V_{3} & =\frac{u-u(S+I)-\alpha \eta I}{1-S-I}=u-\frac{\alpha \eta I}{1-S-I} \\
\mathscr{A}^{*} V_{4} & =-\frac{u-u S-u I-\alpha \eta I}{S+I-(u /(u+\alpha \eta))} \\
& =(u+\alpha \eta)-\frac{\alpha \eta S}{S+I-(u /(u+\alpha \eta))}
\end{aligned}
$$

Thus, according to (54)-(57), we have

$$
\begin{aligned}
\mathscr{A}^{*} V \leq & -G \lambda+\frac{G m I}{1+m I}(1-p) \lambda_{2} \\
& +\frac{G\left[p \lambda_{1}+(1-p) \lambda_{2}\right]}{u}(\alpha \eta+u) I-\frac{u}{S} \\
& -\frac{\alpha \eta I}{1-S-I}-\frac{\alpha \eta S}{S+I-(u /(u+\alpha \eta))}+H,
\end{aligned}
$$

where $\quad H=3 u+\alpha \eta+\left[p \lambda_{1}+(1-p) \lambda_{2}\right]+\left(p^{2} \sigma_{1}^{2}+(1-\right.$ $p)^{2} \sigma_{2}^{2} / 2$ ).

Now, we prove that $\mathscr{A}^{*} V(S, I)<-1$ on the following four domains which are equivalent to $\mathscr{A}^{*} V(S, I)<-1$ on the $E \backslash U$.

(i) $U_{1}=\{(S, I) \in E: 0<I<\varepsilon\}$. If $(S, I) \in U_{1}$, by (47) and (51), we have

$$
\begin{aligned}
\mathscr{A}^{*} V \leq & -G \lambda+\frac{G m I}{1+m I}(1-p) \lambda_{2} \\
& +\frac{G\left[p \lambda_{1}+(1-p) \lambda_{2}\right]}{u}(\alpha \eta+u) I+H \\
& <-G \lambda+G m \varepsilon(1-p) \lambda_{2} \\
& +\frac{G\left[p \lambda_{1}+(1-p) \lambda_{2}\right]}{u}(\alpha \eta+u) \varepsilon+H \\
& <1+(-2) \\
= & -1 .
\end{aligned}
$$

(ii) $U_{2}=\{(S, I) \in E: 0<S<\varepsilon\}$. If $(S, I) \in U_{2}$, using (52), we get

$$
\begin{aligned}
\mathscr{A}^{*} V \leq & -\frac{u}{S}+\frac{G m I}{1+m I}(1-p) \lambda_{2} \\
& +\frac{G\left[p \lambda_{1}+(1-p) \lambda_{2}\right]}{u}(\alpha \eta+u) I+H \\
& <-\frac{u}{\varepsilon}+G m(1-p) \lambda_{2} \\
& +\frac{G\left[p \lambda_{1}+(1-p) \lambda_{2}\right]}{u}(\alpha \eta+u)+H \\
& <-1 .
\end{aligned}
$$

(iii) $U_{3}=\left\{(S, I) \in E: \varepsilon \leq I, 1-\varepsilon^{2}<S+I\right\}$. If $(S, I) \in U_{3}$, in view of (53), we derive

$$
\begin{aligned}
\mathscr{A}^{*} V \leq & -\frac{\alpha \eta I}{1-S-I}+\frac{G m I}{1+m I}(1-p) \lambda_{2} \\
& +\frac{G\left[p \lambda_{1}+(1-p) \lambda_{2}\right]}{u}(\alpha \eta+u) I+H \\
& <-\frac{\alpha \eta}{\varepsilon}+G m(1-p) \lambda_{2} \\
& +\frac{G\left[p \lambda_{1}+(1-p) \lambda_{2}\right]}{u}(\alpha \eta+u)+H \\
& <-1 .
\end{aligned}
$$

(iv) $U_{4}=\left\{(S, I) \in E: \varepsilon \leq S, S+I<(u /(u+\alpha \eta))+\varepsilon^{2}\right\}$. If $(S, I) \in U_{4}$, according to (53), we obtain

$$
\begin{aligned}
\mathscr{A}^{*} V \leq & -\frac{\alpha \eta S}{S+I-(u /(u+\alpha \eta))}+\frac{G m I}{1+m I}(1-p) \lambda_{2} \\
& +\frac{G\left[p \lambda_{1}+(1-p) \lambda_{2}\right]}{u}(\alpha \eta+u) I+H \\
& <-\frac{\alpha \eta}{\varepsilon}+G m(1-p) \lambda_{2} \\
& +\frac{G\left[p \lambda_{1}+(1-p) \lambda_{2}\right]}{u}(\alpha \eta+u)+H \\
& <-1 .
\end{aligned}
$$

In conclusion, for a sufficiently small positive number $\varepsilon$,

$$
\sup _{(S, I) \in E \backslash U} \mathscr{A}^{*} V(S, I)<-1 .
$$

We apply similar arguments to those in [41]; this means that the semigroup is not sweeping from the set $U$. Thus, the semigroup $\{P(t)\}_{t \geq 0}$ is asymptotically stable by Remark 1 .

\section{Dynamics of System (5)}

3.1. The Property of the Positive Solution. The first result is the existence and uniqueness of the positive solution of system (5). 
Theorem 1. System (5) has a unique positive solution $(S(t), I(t))$ on $t \geq 0$ with any initial value $(S(0), I(0)) \in \mathbb{R}_{+}^{2}$ almost surely.

Proof. By system (5), we obtain that

$$
S(t)+I(t) \leq 1+e^{-u t}(S(0)+I(0)-1) \leq K,
$$

where $K=\max \{S(0)+I(0), 1\}$.

Define

$$
V(S, I)=(S-1-\ln S)+(I-1-\ln I) .
$$

Clearly, $V(S, I)$ is nonnegative. Using Itô's formula and the fact $S(t)+I(t) \leq K$, we get

$$
\begin{aligned}
\mathrm{d} V(S, I)= & L V(S, I) \mathrm{d} t+\left[p \sigma_{1}(I-S)\right] \mathrm{d} B_{1}(t) \\
& +\left[\frac{(1-p) \sigma_{2}}{1+m I}(I-S)\right] \mathrm{d} B_{2}(t), \\
L V(S, I)= & u-u S+(1-\alpha) \eta I-\frac{u}{S}+u-\frac{(1-\alpha) \eta I}{S} \\
& +p \lambda_{1} I+\frac{(1-p) \lambda_{2} I}{1+m I}-u I-\eta I+u+\eta+\frac{I^{2}}{2}\left[p^{2} \sigma_{1}^{2}+\frac{(1-p)^{2} \sigma_{2}^{2}}{(1+m I)^{2}}\right]-p \lambda_{1} S \\
& -\frac{(1-p) \lambda_{2} S}{1+m I}+\frac{S^{2}}{2}\left[p^{2} \sigma_{1}^{2}+\frac{(1-p)^{2} \sigma_{2}^{2}}{(1+m I)^{2}}\right] \\
\leq & 3 u+p \lambda_{1} I+\frac{(1-p) \lambda_{2} I}{1+m I}+\eta+\frac{I^{2}}{2}\left[p^{2} \sigma_{1}^{2}+\frac{(1-p)^{2} \sigma_{2}^{2}}{(1+m I)^{2}}\right]+\frac{S^{2}}{2}\left[p^{2} \sigma_{1}^{2}+\frac{(1-p)^{2} \sigma_{2}^{2}}{(1+m I)^{2}}\right] \\
\leq & 3 u+p \lambda_{1} K+\frac{(1-p) \lambda_{2} K}{1+m I}+\eta+\frac{K^{2}}{2}\left[p^{2} \sigma_{1}^{2}+\frac{(1-p)^{2} \sigma_{2}^{2}}{(1+m I)^{2}}\right]+\frac{K^{2}}{2}\left[p^{2} \sigma_{1}^{2}+\frac{(1-p)^{2} \sigma_{2}^{2}}{(1+m I)^{2}}\right] \\
\leq & 3 u+p \lambda_{1} K+(1-p) \lambda_{2} K+\eta+\frac{K^{2}}{2}\left[p^{2} \sigma_{1}^{2}+(1-p)^{2} \sigma_{2}^{2}\right]+\frac{K^{2}}{2}\left[p^{2} \sigma_{1}^{2}+(1-p)^{2} \sigma_{2}^{2}\right] \\
= & 3 u+p \lambda_{1} K+(1-p) \lambda_{2} K+\eta+K^{2} p^{2} \sigma_{1}^{2}+K^{2}(1-p)^{2} \sigma_{2}^{2} \\
:= & K_{0} .
\end{aligned}
$$

Here, $K_{0}$ is a positive constant. Then, refer to [42], and we complete the proof.

\subsection{Asymptotically Stable Stationary Distribution and Ex-} tinction of System (5)

Theorem 2. Denote $(S(t), I(t))$ a solution of system (5) with any initial value $(S(0), I(0)) \in \mathbb{R}_{+}^{2}$; the distribution of $(S(t), I(t))$ has a density $U(t, x, y)$ for every $t>0$. If $R_{0}-\left(\left(p^{2} \sigma_{1}^{2}+(1-p)^{2} \sigma_{2}^{2}\right) / 2(u+\eta)\right)>1$, then there is a unique density $U_{*}(x, y)$ satisfying

$$
\begin{aligned}
& \lim _{t \longrightarrow \infty} \iint_{\mathbb{R}_{+}^{2}}\left|U(t, x, y)-U_{*}(x, y)\right| \mathrm{d} x \mathrm{~d} y=0, \\
& \operatorname{supp}_{*}=\left\{(x, y) \in \mathbb{R}_{+}^{2}: \frac{u}{u+\alpha \eta}<x+y<1\right\}:=E .
\end{aligned}
$$

Proof. The proof of Theorem 2 is the following steps:

Step 1. According to Hö rmander theorem [35], we prove that the transition function of the process $(S(t), I(t))$ is absolutely continuous (see Lemma 1)

Step 2. We verify the positivity of the density of the transition function on $E$ by support theorems [37-39] (refer to Lemma 2)

Step 3. We prove that the Markov semigroup satisfies the Foguel alternative (see Lemmas 3 and 4)

Step 4. Excluding sweeping by verifying there is a Khasminskii function (refer to Lemma 5)

Notice that the above strategies can be proved by Lemmas 1-5; thus, this proof is complete.

Theorem 3. For any initial value $(S(0), I(0)) \in \mathbb{R}_{+}^{2},(S(t)$, $I(t))$ is a solution of system (5). If one of the following conditions holds, the epidemic $I(t)$ becomes extinct with probability one. 
(i)

$$
\begin{aligned}
\sigma_{1}^{2} & \leq \frac{\lambda_{1}}{p}, \\
\sigma_{2}^{2} & \leq \frac{\lambda_{2}}{1-p}, \\
R_{0}-\frac{p^{2} \sigma_{1}^{2}+(1-p)^{2} \sigma_{2}^{2}}{2(u+\eta)} & <1 .
\end{aligned}
$$

(ii)

$$
\frac{\lambda_{1}^{2}}{2 \sigma_{1}^{2}}+\frac{\lambda_{2}^{2}}{2 \sigma_{2}^{2}}<u+\eta
$$

Particularly, if (i) holds,

$$
\begin{aligned}
\limsup _{t \rightarrow \infty} \frac{\ln I(t)}{t} & \leq(u+\eta)\left[R_{0}-1-\frac{p^{2} \sigma_{1}^{2}+(1-p)^{2} \sigma_{2}^{2}}{2(u+\eta)}\right] \\
& <0, \quad \text { a.s. }
\end{aligned}
$$

If (ii) holds,

$$
\limsup _{t \rightarrow \infty} \frac{\ln I(t)}{t} \leq \frac{\lambda_{1}^{2}}{2 \sigma_{1}^{2}}+\frac{\lambda_{2}^{2}}{2 \sigma_{2}^{2}}-u-\eta<0, \quad \text { a.s. }
$$

Proof. If $\sigma_{1}^{2} \leq\left(\lambda_{1} / p\right), \sigma_{2}^{2} \leq\left(\lambda_{2} /(1-p)\right)$, and $R_{0}-\left(\left(p^{2} \sigma_{1}^{2}+\right.\right.$ $\left.\left.(1-p)^{2} \sigma_{2}^{2}\right) / 2(u+\eta)\right)<1$, we obtain

$$
\begin{aligned}
\limsup _{t \rightarrow \infty} \frac{\ln I(t)}{t} \leq & -\frac{p^{2} \sigma_{1}^{2}}{2}\left(1-\frac{\lambda_{1}}{p \sigma_{1}^{2}}\right)^{2}+\frac{\lambda_{1}^{2}}{2 \sigma_{1}^{2}} \\
& -\frac{(1-p)^{2} \sigma_{2}^{2}}{2}\left(1-\frac{\lambda_{2}}{(1-p) \sigma_{2}^{2}}\right)^{2}+\frac{\lambda_{2}^{2}}{2 \sigma_{2}^{2}}-u-\eta \\
= & -\frac{p^{2} \sigma_{1}^{2}}{2}+p \lambda_{1}-\frac{(1-p)^{2} \sigma_{2}^{2}}{2}+(1-p) \lambda_{2}-u-\eta \\
= & (u+\eta)\left(R_{0}-1-\frac{p^{2} \sigma_{1}^{2}+(1-p)^{2} \sigma_{2}^{2}}{2(u+\eta)}\right),
\end{aligned}
$$

and notice that $R_{0}-\left(\left(p^{2} \sigma_{1}^{2}+(1-p)^{2} \sigma_{2}^{2}\right) / 2(u+\eta)\right)<1$; then,

$$
(u+\eta)\left(R_{0}-1-\frac{p^{2} \sigma_{1}^{2}+(1-p)^{2} \sigma_{2}^{2}}{2(u+\eta)}\right)<0 .
$$

Then,

$$
\limsup _{t \rightarrow \infty} \frac{\ln I(t)}{t}<0, \quad \text { a.s. }
$$

If $\left(\lambda_{1}^{2} / 2 \sigma_{1}^{2}\right)+\left(\lambda_{2}^{2} / 2 \sigma_{2}^{2}\right)<u+\eta$, we easily get that

$$
\begin{aligned}
\limsup _{t \rightarrow \infty} \frac{\ln I(t)}{t}= & \limsup _{t \rightarrow \infty}\left[\frac{p \lambda_{1} \int_{0}^{t} S(s) \mathrm{d} s}{t}-\frac{p^{2} \sigma_{1}^{2} \int_{0}^{t} S^{2}(s) \mathrm{d} s}{2 t}\right] \\
& +\limsup _{t \rightarrow \infty}\left[\frac{(1-p) \lambda_{2} \int_{0}^{t}(S(s) /(1+m I(s))) \mathrm{d} s}{t}-u-\eta\right] \\
& -\limsup _{t \rightarrow \infty}\left[\frac{(1-p)^{2} \sigma_{2}^{2} \int_{0}^{t}(S(s) /(1+m I(s)))^{2} \mathrm{~d} s}{2 t}\right] \\
& +\limsup _{t \rightarrow \infty}\left[\frac{M_{1}(t)}{t}+\frac{M_{2}(t)}{t}\right] \\
\leq & \limsup _{t \rightarrow \infty}\left[\frac{\left.p \lambda_{1} \int_{0}^{t} S(s) \mathrm{d} s\right]}{t}\right] \\
& -\limsup _{t \longrightarrow \infty}\left[\frac{p^{2} \sigma_{1}^{2}}{2}\left(\frac{\int_{0}^{t} S^{2}(s) \mathrm{d} s}{t}\right)^{2}\right]
\end{aligned}
$$




$$
\begin{aligned}
& +\underset{t \rightarrow \infty}{\limsup _{\longrightarrow}}\left[\frac{(1-p) \lambda_{2} \int_{0}^{t}(S(s) /(1+m I(s))) \mathrm{d} s}{t}-u-\eta\right] \\
& -\limsup _{t \longrightarrow \infty}\left[\frac{(1-p)^{2} \sigma_{2}^{2}}{2}\left(\frac{\int_{0}^{t}(S(s) /(1+m I(s)) \mathrm{d} s}{t}\right)\right] \\
& \left.=-\frac{p^{2} \sigma_{1}^{2}}{2} \liminf _{t \longrightarrow \infty}\left[\frac{\int_{0}^{t} S(s) \mathrm{d} s}{t}-\frac{\lambda_{1}}{p \sigma_{1}^{2}}\right)^{2}+\frac{\lambda_{1}^{2}}{2 \sigma_{1}^{2}}\right] \\
& -\frac{(1-p)^{2} \sigma_{2}^{2}}{2} \liminf _{t \longrightarrow \infty} J^{2}+\frac{\lambda_{2}^{2}}{2 \sigma_{2}^{2}}-u-\eta \\
& \leq \frac{\lambda_{1}^{2}}{2 \sigma_{1}^{2}}+\frac{\lambda_{2}^{2}}{2 \sigma_{2}^{2}}-u-\eta \\
& <0, \quad \text { a.s. },
\end{aligned}
$$

where $\quad J=\left(\int_{0}^{t}(S(s) /(1+m I(s))) \mathrm{d} s / t\right)-\left(\lambda_{2} /(1-p) \sigma_{2}^{2}\right)$. This proof is completed.

Remark 4. $R_{0}-\left(\left(p^{2} \sigma_{1}^{2}+(1-p)^{2} \sigma_{2}^{2}\right) / 2(u+\eta)\right)$ is the threshold of system (5) when $\sigma_{1}^{2} \leq\left(\lambda_{1} / p\right)$ and $\sigma_{2}^{2} \leq$ $\left(\lambda_{2} /(1-p)\right)$.

\section{Simulations and Conclusion}

Now, in order to illustrate the analytical results, we make some numerical simulations. For systems (3) and (4), the parameter values are $u=0.2, \alpha=0.4, p=0.6, \lambda_{1}=$ $0.5, \lambda_{2}=0.6, \eta=0.2$, and $m=0.2 ; \quad$ then, $\quad R_{0}=\left(\left(p \lambda_{1}+\right.\right.$ $\left.\left.(1-p) \lambda_{2}\right) /(u+\eta)\right)=1.35>1$.

We employ the following discrete equations:

$$
\left\{\begin{aligned}
S_{k}+1 & =\left[u-u S_{k}-p \lambda_{1} S_{k} I_{k}-(1-p) \frac{\lambda_{2} S_{k} I_{k}}{1+m I_{k}}\right] \Delta t+\left[(1-\alpha) \eta I_{k}\right] \Delta t-\left[(1-p) \frac{\sigma_{2} S_{k} I_{k}}{1+m I_{k}}\right] \sqrt{\Delta t} \xi_{k} \\
& -\left[(1-p) \frac{\sigma_{2}^{2} S_{k} I_{k}}{2\left(1+m I_{k}\right)}\right]\left(\xi_{k}^{2}-1\right) \Delta t+S_{k}-\left(p \sigma_{1} S_{k} I_{k}\right) \sqrt{\Delta t} \xi_{k}-\left(\frac{p \sigma_{1}^{2} S_{k} I_{k}}{2}\right)\left(\xi_{k}^{2}-1\right) \Delta t, \\
I_{k}+1 & =\left[p \lambda_{1} S_{k} I_{k}+(1-p) \frac{\lambda_{2} S_{k} I_{k}}{1+m I_{k}}-u I_{k}-\eta I_{k}\right] \Delta t+\left(p \sigma_{1} S_{k} I_{k}\right) \sqrt{\Delta t} \xi_{k}+\left(\frac{p \sigma_{1}^{2} S_{k} I_{k}}{2}\right)\left(\xi_{k}^{2}-1\right) \Delta t \\
+ & I_{k}+\left[(1-p) \frac{\sigma_{2} S_{k} I_{k}}{1+m I_{k}}\right] \sqrt{\Delta t} \xi_{k}+\left[(1-p) \frac{\sigma_{2}^{2} S_{k} I_{k}}{2\left(1+m I_{k}\right)}\right]\left(\xi_{k}^{2}-1\right) \Delta t, \\
R_{k}+1 & =R_{k}+\left(\alpha \eta I_{k}-u R_{k}\right) \Delta t,
\end{aligned}\right.
$$

where $\xi_{k}(k=1,2, \ldots, n)$ are $N(0,1)$-distributed independent Gaussian random variables.

Example 1. Let $\sigma_{1}=0.2$ and $\sigma_{2}=0.3$; then,

$$
R_{0}-1=0.35>0.0065=\frac{p^{2} \sigma_{1}^{2}+(1-p)^{2} \sigma_{2}^{2}}{2(u+\eta)},
$$

which satisfies the condition in Theorem 2; this means that epidemic $I(t)$ is persistent for long time. Figures 1 (a) and 1 (b) are the paths of individuals in deterministic model (3) and stochastic model (4), respectively. Figure 1(c) is the phase portrait of Figures 1(a) and 1(b). Figures 2(a)2(c) are the distribution diagrams of $S, I$ and $R$, respectively. 

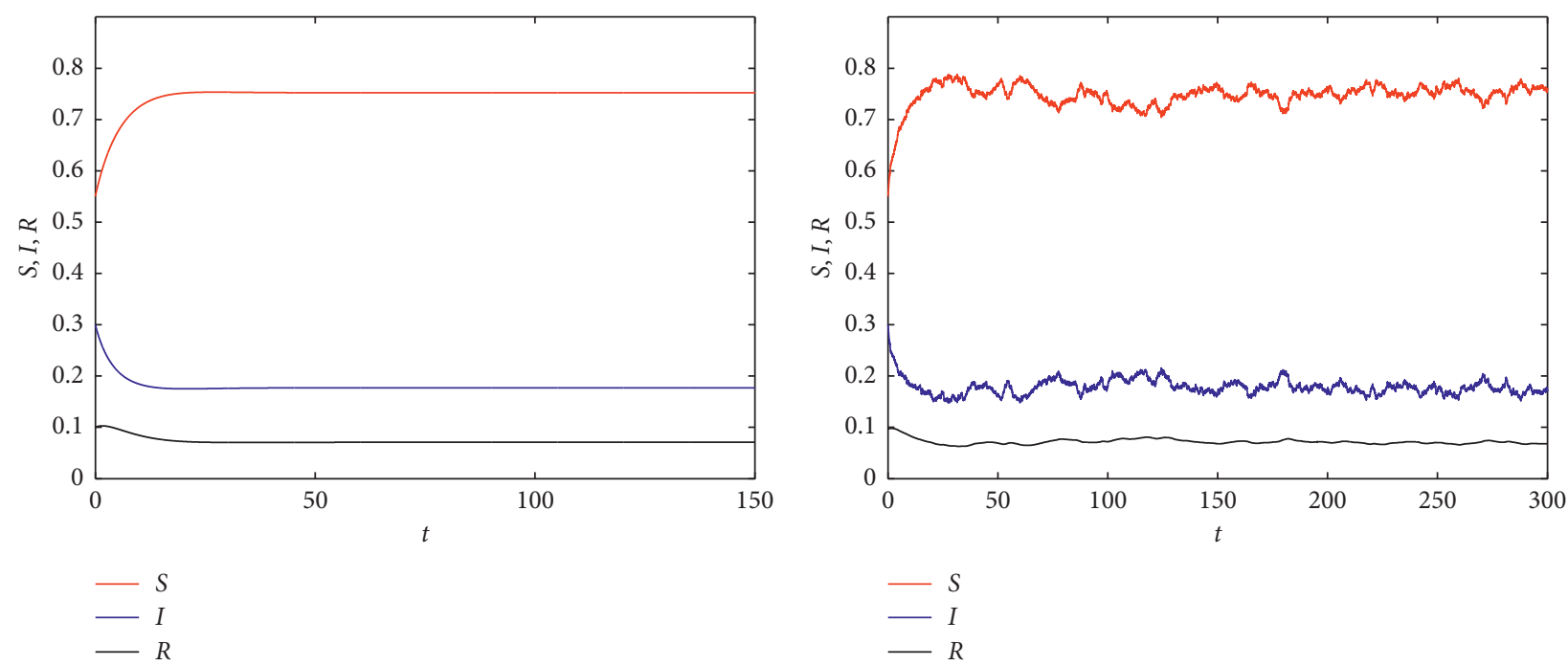

(a)

(b)

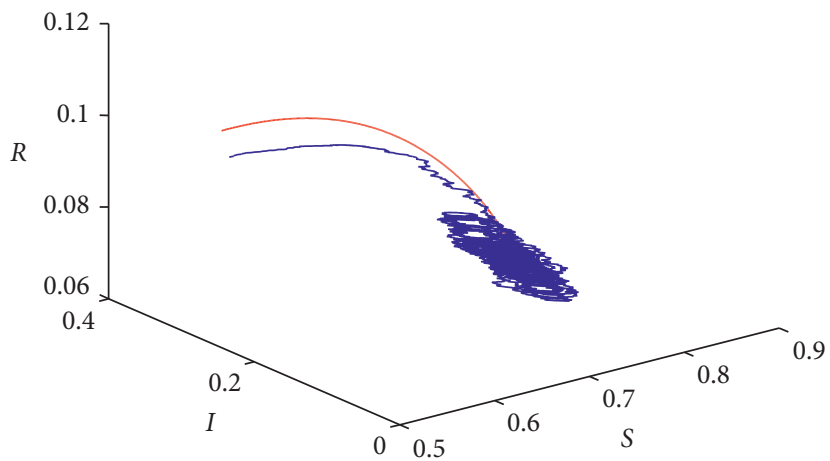

- Stochastic model

Deterministic model

(c)

FiguRe 1: (a) Deterministic system, (b) corresponding stochastic systems of (a), and (c) the phase portrait of (b). (a) $\sigma_{1}=0, \sigma_{2}=0$; (b) $\sigma_{1}=0.1, \sigma_{2}=0.1$.

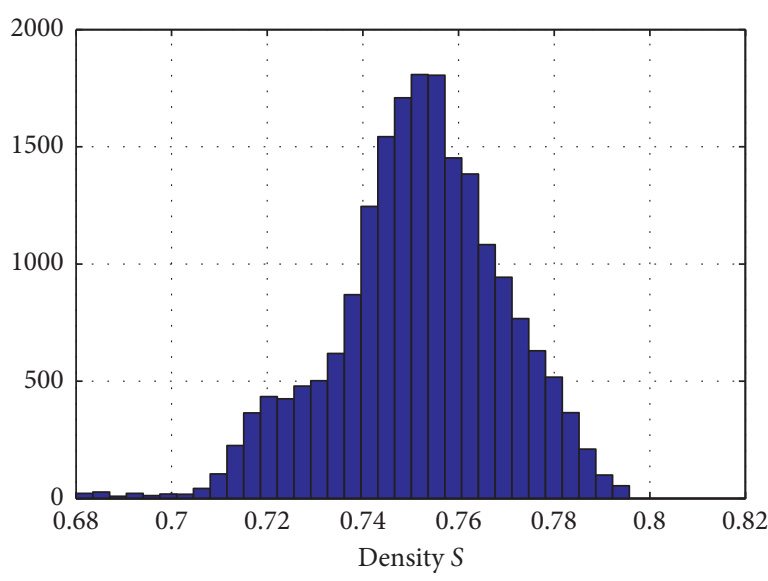

(a)

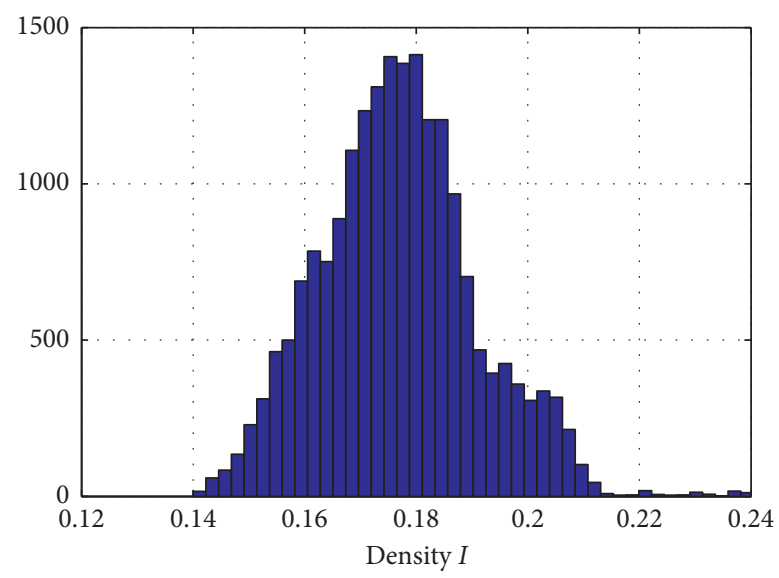

(b)

Figure 2: Continued. 


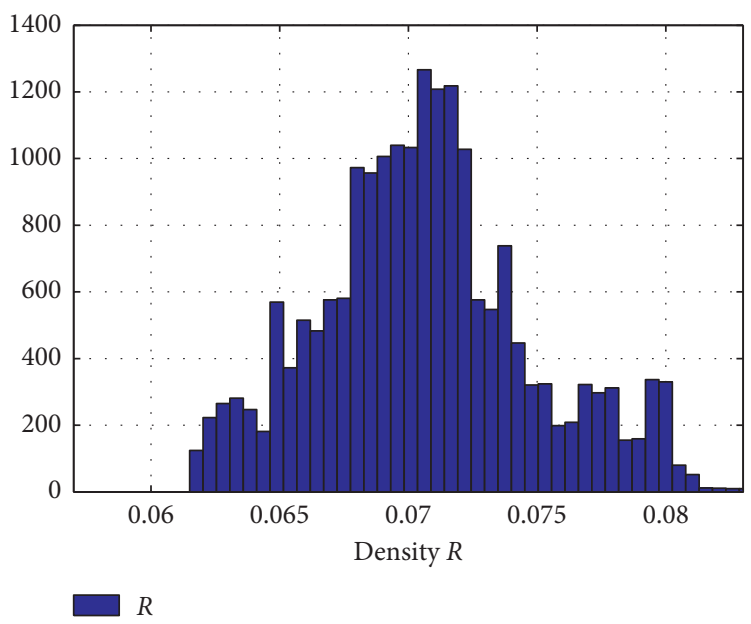

(c)

Figure 2: $\left(\mathrm{a}, \mathrm{b}\right.$, and $\mathrm{c}$ ) The distribution diagram of $S, I$, and $R$, respectively. $\sigma_{1}=0.1, \sigma_{2}=0.1$.

Example 2. Case 1: let $\sigma_{1}=0.95$ and $\sigma_{2}=0.95$; then,

$$
\frac{\lambda_{1}^{2}}{2 \sigma_{1}^{2}}+\frac{\lambda_{2}^{2}}{2 \sigma_{2}^{2}}=0.33795<0.4=u+\eta,
$$

which satisfies condition (ii) in Theorem 3; this means that epidemic $I(t)$ becomes extinct with probability one. Figures 3(a) and 3(b) show the paths of individuals in deterministic model (3) and stochastic model (4), respectively. Figure $3(\mathrm{c})$ shows the phase portrait of Figures 3(a) and 3(b).

Case 2: let $\sigma_{1}=0.85$ and $\sigma_{2}=0.85$; then,

$$
\begin{aligned}
\sigma_{1}^{2} & =0.7225 \leq 0.8333=\frac{\lambda_{1}}{p}, \\
\sigma_{2}^{2} & =0.7225 \leq 1.5=\frac{\lambda_{2}}{1-p}, \\
R_{0}-1 & =0.35<0.46962=\frac{p^{2} \sigma_{1}^{2}+(1-p)^{2} \sigma_{2}^{2}}{2(u+\eta)},
\end{aligned}
$$

which satisfies condition (i) in Theorem 3; this means that epidemic $I(t)$ becomes extinct with probability one. Figures 4(a) and 4(b) show the paths of individuals in deterministic model (3) and stochastic model (4), respectively. Figure $4(\mathrm{c})$ is the phase portrait of Figures $4(\mathrm{a})$ and $4(\mathrm{~b})$.

We have studied a stochastic SIR epidemic system affected by mixed nonlinear incidence rates in this paper. Using Markov semigroup theory and Fokker-Planck equation, we proved that the value of $R_{0}-\left(\left(p^{2} \sigma_{1}^{2}+\right.\right.$ $\left.\left.(1-p)^{2} \sigma_{2}^{2}\right) / 2(u+\eta)\right)$ is the threshold of system (5) when $\sigma_{1}^{2} \leq\left(\lambda_{1} / p\right)$ and $\sigma_{2}^{2} \leq\left(\lambda_{2} /(1-p)\right)$. If $R_{0}-\left(\left(p^{2} \sigma_{1}^{2}+(1-\right.\right.$ $\left.\left.p)^{2} \sigma_{2}^{2}\right) / 2(u+\eta)\right)>1$, the epidemic is persistent, and there is a unique asymptotically stable stationary distribution. If $R_{0}-\left(\left(p^{2} \sigma_{1}^{2}+(1-p)^{2} \sigma_{2}^{2}\right) / 2(u+\eta)\right)<1$, the sufficient conditions for disease extinction are established, that is, if $\sigma_{1}^{2} \leq\left(\lambda_{1} / p\right)$ and $\sigma_{2}^{2} \leq\left(\lambda_{2} / p\right)$, then $\lim \sup _{t \rightarrow \infty}(\ln I(t) / t)<0 ;$ this means that the epidemic becomes extinct with probability one. Moreover, if $\left(\lambda_{1}^{2} / 2 \sigma_{1}^{2}\right)+\left(\lambda_{2}^{2} / 2 \sigma_{2}^{2}\right)<u+\eta$, the epidemic is also extinct.

By the conclusion of Theorem 1, it is worthy to point out that two different incidence rates which are considered at the same time will not destroy a great property that existence and uniqueness of the positive solution. From Theorems 2 and 3, we know that when two infection rates are considered at the same time, in addition to the values of $\sigma_{1}$ and $\sigma_{2}$, the value of $p$ also affects the threshold value $R_{0}-\left(\left(p^{2} \sigma_{1}^{2}+(1-p)^{2} \sigma_{2}^{2}\right) / 2(u+\eta)\right)$. In addition, from the sufficient conditions for disease extinction, parameter $p$ is an important variable related to the extinction of the population. Especially, when the parameters $p=1$ and $\alpha=1$, system (3) becomes (1) in [12], and system (3) is similar to (2) in [13] when the parameters $p=0$ and $\alpha=1$. To some extent, our model is more realistic than considering the epidemic affected by a single nonlinear incidence rate.

Since the proposed system is degenerate, we use Markov semigroup theory to study the stationary distribution and ergodicity of the system, and we can also study color noise and other noises in the future. For other noises, this method is feasible as long as it conforms to the relevant properties of Gaussian white noise. In addition, two aspects can be used as a guide for further research. First of all, we can investigate some other systems, for example, one can consider the systems with the impulsive perturbation effects $[43,44]$. In addition, it is interesting to study the chemostat as well as population dynamics systems [45-51]. Notice that it is a meaningful question to investigate whether the way used in this article is applied to other epidemic systems. These questions are worthy of further study. 

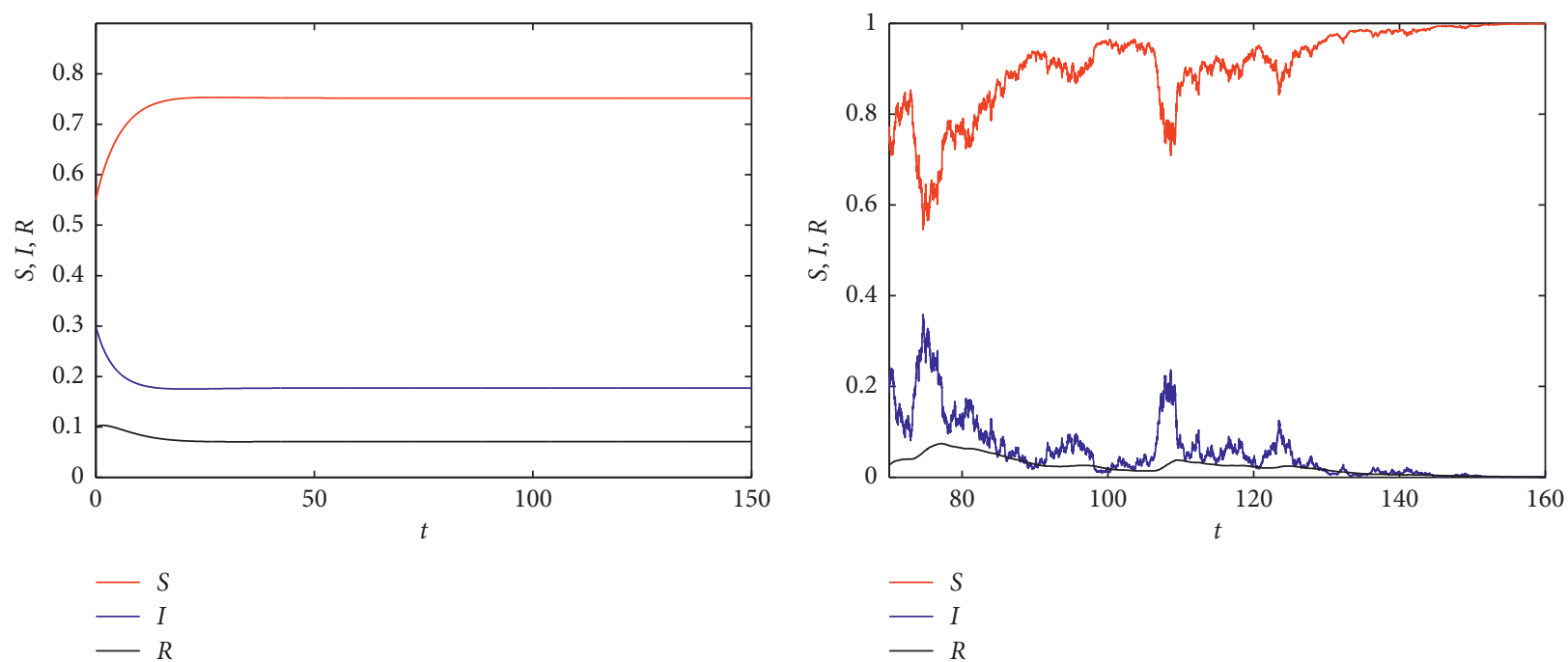

(a)

(b)

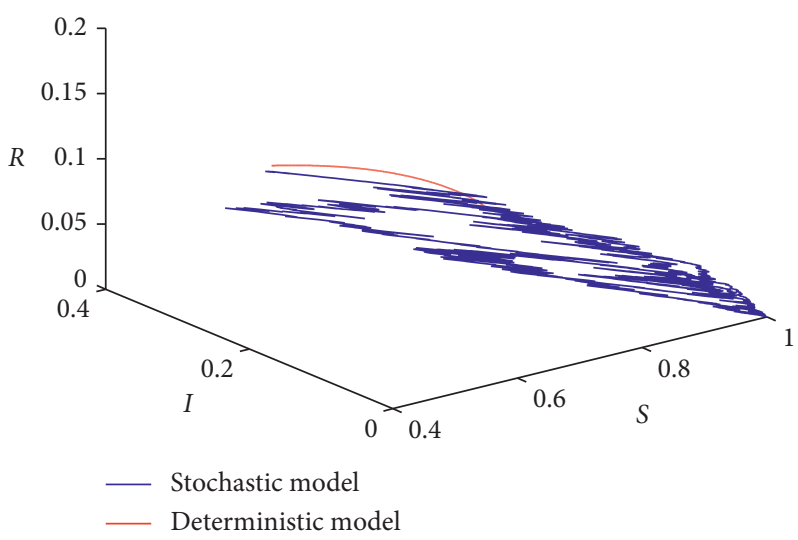

(c)

FIGURE 3: (a) Deterministic system, (b) corresponding stochastic systems of (a), and (c) the phase portrait of (b). (a) $\sigma_{1}=0, \sigma_{2}=0$; (b) $\sigma_{1}=0.95, \sigma_{2}=0.95$.
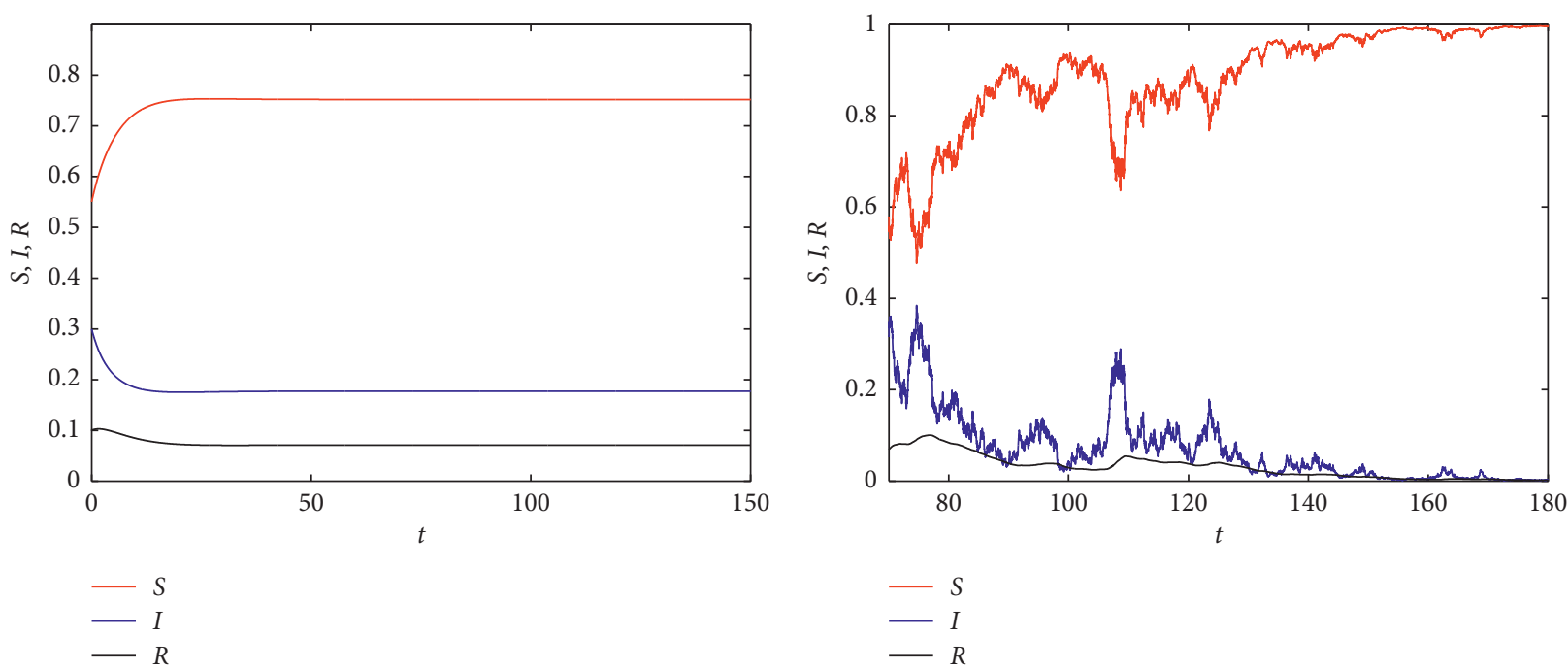

(a)

(b)

Figure 4: Continued. 


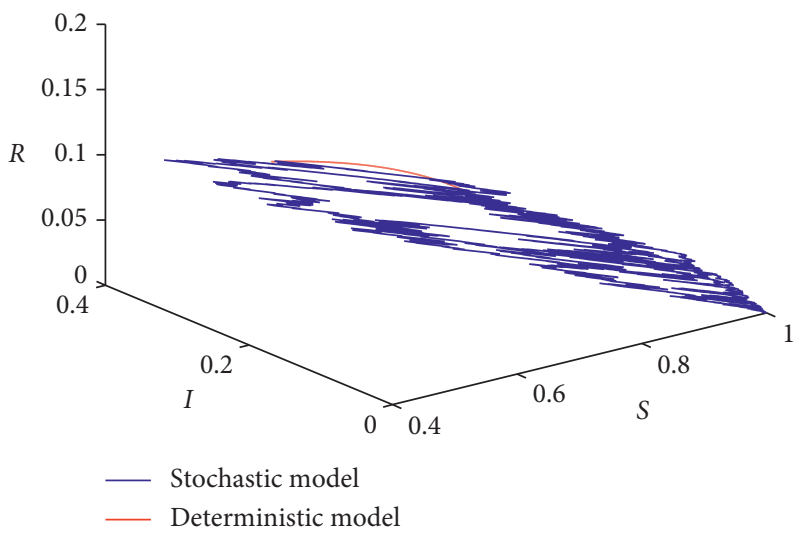

(c)

Figure 4: (a) Deterministic system, (b) corresponding stochastic systems of (a), and (c) the phase portrait of (b). (a) $\sigma_{1}=0, \sigma_{2}=0$; (b) $\sigma_{1}=0.85, \sigma_{2}=0.85$.

\section{Data Availability}

No data were used to support this study.

\section{Conflicts of Interest}

The authors declare that they have no conflicts of interest.

\section{Authors' Contributions}

All authors read and approved the final manuscript.

\section{Acknowledgments}

This work was supported by the Research Fund for the Taishan Scholar Project of Shandong Province of China, Shandong Provincial Natural Science Foundation of China (ZR2019MA003).

\section{References}

[1] W. O. Kermack and A. G. McKendrick, "Contributions to the mathematical theory of epidemics-I," Bulletin of Mathematical Biology, vol. 115, pp. 700-721, 1927.

[2] A. Lahrouz and L. Omari, "Extinction and stationary distribution of a stochastic SIRS epidemic model with non-linear incidence," Statistics \& Probability Letters, vol. 83, no. 4, pp. 960-968, 2013.

[3] Y. Cai, Y. Kang, M. Banerjee, and W. Wang, "A stochastic SIRS epidemic model with infectious force under intervention strategies," Journal of Differential Equations, vol. 259, no. 12, pp. 7463-7502, 2015.

[4] D. Li, J. a. Cui, M. Liu, and S. Liu, "The evolutionary dynamics of stochastic epidemic model with nonlinear incidence rate," Bulletin of Mathematical Biology, vol. 77, no. 9, pp. 1705-1743, 2015.

[5] R. Rifhat, Q. Ge, and Z. Teng, "The dynamical behaviors in a stochastic SIS epidemic model with nonlinear incidence," Comput. Math. Methods Med.vol. 2016, Article ID 5218163, 14 pages, 2016.

[6] Z. Teng and L. Wang, "Persistence and extinction for a class of stochastic SIS epidemic models with nonlinear incidence rate," Physica A: Statistical Mechanics and Its Applications, vol. 451, pp. 507-518, 2016.

[7] X. Wang, Z. Wang, and H. Shen, "Dynamical analysis of a discrete-time SIS epidemic model on complex networks," Applied Mathematics Letters, vol. 94, pp. 292-299, 2019.

[8] X. Wang, Z. Wang, X. Huang, and Y. Li, "Dynamic analysis of a delayed fractional-order SIR model with saturated incidence and treatment functions," International Journal of Bifurcation and Chaos, vol. 28, no. 14, Article ID 1850180, 2018.

[9] H. Qi, X. Meng, and Z. Chang, "Markov semigroup approach to the analysis of a nonlinear stochastic plant disease model," Electronic Journal of Differential Equations, vol. 2019, no. 116, pp. 1-19, 2019.

[10] K. Liu, T. Zhang, and L. Chen, "State-dependent pulse vaccination and therapeutic strategy in an SI epidemic model with nonlinear incidence rate," Computational and Mathematical Methods in Medicine, vol. 2019, 10 pages, 2019.

[11] Y. Zhang, K. Fan, S. Gao, Y. Liu, and S. Chen, "Ergodic stationary distribution of a stochastic SIRS epidemic model incorporating media coverage and saturated incidence rate," Physica A: Statistical Mechanics and Its Applications, vol. 514, pp. 671-685, 2019.

[12] H. W. Hethcote, "Qualitative analyses of communicable disease models," Mathematical Biosciences, vol. 28, no. 3-4, pp. 335-356, 1976.

[13] Q. Yang, D. Jiang, N. Shi, and C. Ji, "The ergodicity and extinction of stochastically perturbed SIR and SEIR epidemic models with saturated incidence," Journal of Mathematical Analysis and Applications, vol. 388, no. 1, pp. 248-271, 2012.

[14] Q. Liu, D. Jiang, T. Hayat, and A. Alsaedi, "Threshold dynamics of a stochastic SIS epidemic model with nonlinear incidence rate," Physica A: Statistical Mechanics and Its Applications, vol. 526, p. 120946, 2019.

[15] G. Liu, H. Qi, Z. Chang, and X. Meng, "Asymptotic stability of a stochastic May mutualism system," Computers \& Mathematics with Applications, vol. 79, no. 3, pp. 735-745, 2020.

[16] M. Liu, "Dynamics of a stochastic regime-switching predatorprey model with modified Leslie-Gower Holling-type II schemes and prey harvesting," Nonlinear Dynamics, vol. 96, no. 1, pp. 417-442, 2019.

[17] M. Liu, J. Yu, and P. S. Mandal, "Dynamics of a stochastic delay competitive model with harvesting and Markovian 
switching," Applied Mathematics and Computation, vol. 337, pp. 335-349, 2018.

[18] X. Yu, S. Yuan, and T. Zhang, "Persistence and ergodicity of a stochastic single species model with Allee effect under regime switching," Communications in Nonlinear Science and Numerical Simulation, vol. 59, pp. 359-374, 2018.

[19] X. Mao, G. Marion, and E. Renshaw, "Environmental Brownian noise suppresses explosions in population dynamics," Stochastic Processes and Their Applications, vol. 97, no. 1, pp. 95-110, 2002.

[20] L. J. S. Allen and E. J. Allen, "A comparison of three different stochastic population models with regard to persistence time," Theoretical Population Biology, vol. 64, no. 4, pp. 439-449, 2003.

[21] F. Li, S. Zhang, and X. Meng, "Dynamics analysis and numerical simulations of a delayed stochastic epidemic model subject to a general response function," Computational and Applied Mathematics, vol. 38, no. 2, p. 95, 2019.

[22] W. Zhao, J. Liu, M. Chi, and F. Bian, "Dynamics analysis of stochastic epidemic models with standard incidence," Advances in Difference Equations, vol. 2019, no. 1, p. 22, 2019.

[23] H. Qi, L. Liu, and X. Meng, "Dynamics of a nonautonomous stochastic SIS epidemic model with double epidemic hypothesis," Complexity, vol. 2017, Article ID 4861391, 14 pages, 2017.

[24] Y. Zhao, T. Zhang, and W. Zhang, “Asynchronous $H_{\infty}$ control for uncertain singular stochastic Markov jump systems with multiplicative noise based on hidden Markov mode," Journal of the Franklin Institute, 2020.

[25] H. Qi, X. Meng, and T. Feng, "Dynamics analysis of a stochastic non-autonomous one-predator-two-prey system with Beddington-DeAngelis functional response and impulsive perturbations," Advances in Difference Equations, vol. 2019, no. 1, p. 235, 2019.

[26] F. Wang, B. Chen, Y. Sun, and C. Lin, "Finite time control of switched stochastic nonlinear systems," Fuzzy Sets and Systems, vol. 365, pp. 140-152, 2019.

[27] Y. Zhao, T. Zhang, Y. Fu, and L. Ma, "Finite-time stochastic $H_{\infty}$ control for singular markovian jump systems with $-(x, v)$ dependent noise and generally uncertain transition rates," IEEE Access, vol. 7, pp. 64812-64826, 2019.

[28] F. Wang, Z. Liu, Y. Zhang, and C. L. P. Chen, "Adaptive finitetime control of stochastic nonlinear systems with actuator failures," Fuzzy Sets and Systems, vol. 374, pp. 170-183, 2019.

[29] W. Wang, Y. Cai, Z. Ding, and Z. Gui, "A stochastic differential equation SIS epidemic model incorporating Ornstein-Uhlenbeck process," Physica A: Statistical Mechanics and Its Applications, vol. 509, pp. 921-936, 2018.

[30] R. Rudnicki, "Long-time behaviour of a stochastic preypredator model," Stochastic Processes and Their Applications, vol. 108, no. 1, pp. 93-107, 2003.

[31] R. Rudnicki and K. Pichór, "Influence of stochastic perturbation on prey-predator systems," Mathematical Biosciences, vol. 206, no. 1, pp. 108-119, 2007.

[32] R. Rudnicki, K. Pichór, and M. Tyran-Kamińska, Markov Semigroups and Their Applications, Dynamics of Dissipation, pp. 215-238, Springer, Berlin, Germany, 2002.

[33] K. Pichór and R. Rudnicki, "Continuous Markov semigroups and stability of transport equations," Journal of Mathematical Analysis and Applications, vol. 249, no. 2, pp. 668-685, 2000.

[34] A. Lasota and M. C. Mackey, Chaos, Fractals, and Noise: Stochastic Aspects of Dynamics, Vol. 97, Springer Science \& Business Media, Berlin, Germany, 2013.
[35] D. R. Bell, The Malliavin Calculus, Dover Publications, New York, USA, 2006.

[36] Y. Lin and D. Jiang, "Long-time behaviour of a perturbed SIR model by white noise," Discrete \& Continuous Dynamical Systems-B, vol. 18, no. 7, pp. 1873-1887, 2013.

[37] S. Aida, S. Kusuoka, and D. Strook, "On the support of Wiener functionals, Asymptotic problems in probability theory: wiener functionals and asymptotic," in Pitman Research Notes in Mathematics Series, vol. 284, pp. 3-34, Longman Scientific \& Technical, New York, USA, 1993.

[38] D. W. Stroock and S. R. S. Varadhan, "On the support of diffusion processes with applications to the strong maximum principle," Proceedings of the Sixth Berkeley Symposium on Mathematical Statistics and Probability, vol. 3, pp. 333-359, 1972.

[39] G. B. Arous and R. Léandre, "Décroissance exponentielle du noyau de la chaleur sur la diagonale (II)," Probability Theory and Related Fields, vol. 90, no. 3, pp. 377-402, 1991.

[40] X. Mao, Stochastic Differential Equations and Applications, Horwood, Chichester, UK, 2007.

[41] K. Pichór and R. Rudnicki, "Stability of Markov semigroups and applications to parabolic systems," Journal of Mathematical Analysis and Applications, vol. 215, no. 1, pp. 56-74, 1997.

[42] A. Gray, D. Greenhalgh, L. Hu, X. Mao, and J. Pan, "A stochastic differential equation SIS epidemic model," SIAM Journal on Applied Mathematics, vol. 71, no. 3, pp. 876-902, 2011.

[43] X. Yu, S. Yuan, and T. Zhang, "Survival and ergodicity of a stochastic phytoplankton-zooplankton model with toxinproducing phytoplankton in an impulsive polluted environment," Applied Mathematics and Computation, vol. 347, pp. 249-264, 2019.

[44] G. Liu, Z. Chang, and X. Meng, "Asymptotic analysis of impulsive dispersal predator-prey systems with Markov switching on finite-state space," Journal of Function Spaces, vol. 2019, Article ID 8057153, 18 pages, 2019.

[45] Z. Shi, H. Cheng, Y. Liu, and Y. Wang, "Optimization of an integrated feedback control for a pest management predatorprey model," Mathematical Biosciences and Engineering, vol. 16, no. 6, pp. 7963-7981, 2019.

[46] M. Chi and W. Zhao, "Dynamical analysis of two-microorganism and single nutrient stochastic chemostat model with Monod-Haldane response function," Complexity, vol. 2019, Article ID 8719067, 13 pages, 2019.

[47] Z. Jiang, X. Bi, T. Zhang et al., "Global Hopf bifurcation of a delayed phytoplankton-zooplankton system considering toxin producing effect and delay dependent coefficient," Mathematical Biosciences and Engineering, vol. 16, no. 5, pp. 3807-3829, 2019.

[48] R. Yan and S. Sun, "Stochastic characteristics of a chemostat model with variable yield," Physica A: Statistical Mechanics and its Applications, vol. 537, Article ID 122681, 2020.

[49] T. Feng, X. Meng, T. Zhang, and Z. Qiu, "Analysis of the predator-prey interactions: a stochastic model incorporating disease invasion," Qualitative Theory of Dynamical Systems, vol. 19, no. 2, p. 55, 2020.

[50] Y. Mu, Z. Li, H. Xiang, and H. Wang, "Dynamical analysis of a stochastic multispecies turbidostat model," Complexity, vol. 2019, Article ID 4681205, 18 pages, 2019.

[51] Z. Shi, Y. Li, and H. Cheng, "Dynamic analysis of a pest management smith model with impulsive state feedback control and continuous delay," Mathematics, vol. 7, no. 7, p. 591, 2019. 